Frank Decker

\title{
Direktwahl des Premierministers. Das Scheitern des präsidentiell-parlamentarischen Systems in Israel
}

\author{
1. Zur theoretischen Kontroverse um präsidentiell-parlamentarische \\ "Mischsysteme"
}

Am Ausgangspunkt der vergleichenden Institutionenanalyse demokratischer Gesellschaften steht traditionell die Unterscheidung zwischen dem parlamentarischen und präsidentiellen Regierungssystemtypus. Die Zentralität dieser Unterscheidung bedarf keiner besonderen Begründung, thematisiert sie doch das Verhältnis der beiden »Kerngewalten « des politischen Systems - Exekutive und Legislative -, die dessen demokratische Substanz zuallererst verkörpern. Die Terminologie gibt dabei zugegebenermaßen zu Missverständnissen Anlass. Nicht nur, dass ein wachsender Teil der Rechtsetzung inzwischen in Verordnungsform unmittelbar durch die Exekutiven erfolgt; die Regierungen nehmen heute auch auf die förmlichen Gesetzgebungsprozesse so starken Einfluss, dass das hergebrachte Verständnis von »ausführender (= exekutiver) und »gesetzgebender« (= legislativer) Gewalt als überholt gelten muss. ${ }^{1}$ Des Weiteren besagen die Bezeichnungen "parlamentarisch « und "präsidentiell« nichts über die tatsächliche Machtverteilung zwischen den Gewalten. Die Vorstellung, dass im ersten Falle das Parlament und im letzten Falle der Präsident die einflussreichere Institution sei, übersieht, dass die Legislativen gerade in den parlamentarisch genannten Regierungssystemen an eigenständiger Gestaltungsmacht eingebüßt haben. Typologisierungen der parlamentarischen und präsidentiellen Systeme tun deshalb gut daran, es zunächst bei formellen Unterscheidungskriterien zu belassen und diese mit der Regierungswirklichkeit nicht zu verquicken.

Beginnend mit Douglas Verney ${ }^{2}$ sind von Vertretern der politikwissenschaftlichen Komparatistik zahlreiche Arbeiten vorgelegt worden, die sich um eine solche Typologie bemühen. Methodisch lassen sich dabei zwei »Schulen« unterscheiden. Die einen Autoren wenden ein katalogartiges Verfahren an, versuchen also, verschiedene Merkmale der jeweiligen Systemtypen zu einem möglichst vollständigen »Syndrom « zusammenzufassen. So werden als Merkmale des präsidentiellen Systems für gewöhnlich genannt: die Volkswahl des Präsidenten / chief executive auf eine bestimmte Amtszeit, die formelle Unabhängigkeit von Exekutive und Legislative (das Parlament kann den Präsidenten nicht abberufen, der Präsident das Parlament nicht auflösen) und die Ver-

1 Vgl. Klaus von Beyme, Die parlamentarische Demokratie. Entstehung und Funktionsweise 1789 - 1999, 3., völlig neubearbeitete Auflage, Opladen 1999.

2 Douglas V. Verney, The Analysis of Political Systems, London 1959. 
einigung der Funktion des Regierungschefs mit derjenigen des Staatsoberhaupts (geschlossene Exekutive). Umgekehrt gelten als Merkmale des parlamentarischen Systems: die legitimatorische Verschränkung von Exekutive und Legislative (Regierung bzw. Regierungschef gehen aus dem Parlament hervor und können von diesem abberufen werden; die Exekutive wiederum verfügt über die Möglichkeit, das Parlament aufzulösen) und die zweigeteilte Exekutive (neben den Regierungschef tritt ein Monarch oder gewählter Präsident, der die Funktion des Staatsoberhaupts ausübt).

Das Problem dieser Vorgehensweise liegt darin, dass sie die Merkmale lediglich aneinander reiht, ohne sie näher zu qualifizieren. So ist z.B. nicht einzusehen, weshalb es sich bei der ein- oder zweiteiligen Exekutive um ein zwingendes Unterscheidungskriterium zwischen präsidentiellem und parlamentarischen System handeln soll. Die Herausbildung der zweigeteilten Exekutive ist vor dem geschichtlichen Entstehungshintergrund der parlamentarischen Systeme zu sehen, bei denen sich die demokratisch verantwortlichen Regierungen von der monarchischen Gewalt erst emanzipieren mussten. Nach vollzogener Parlamentarisierung konnte die Krone deshalb als politisch machtlose Institution in den meisten europäischen Ländern weiter existieren, während sie in den verspäteten Demokratien - Frankreich, Italien und Deutschland - durch ein republikanisches Staatsoberhaupt ersetzt wurde. ${ }^{3}$ In parlamentarischen Systemen, die - wie Deutschland und Österreich - zugleich Bundesstaaten sind, ist es zudem nicht ungewöhnlich, dass auf der gliedstaatlichen Ebene der jeweilige Regierungschef die Aufgaben eines Staatsoberhaupts mit übernimmt. Auf der gesamtstaatlichen Ebene bleibt dieses Modell allerdings die Ausnahme; allein Südafrika hat sich nach seinem Austritt aus dem britischen Commonwealth dafür entschieden, die beiden Funktionen zusammenzuführen. ${ }^{4}$

Das Unterscheidungskriterium geschlossene / zweiteilige Exekutive ist auch deshalb problematisch, weil in einem System mit parlamentarisch verantwortlichem Regierungschef und gewähltem Präsidenten letzterer nicht auf die repräsentativ-zeremoniellen Funktionen eines Staatsoberhauptes beschränkt bleiben muss, sondern selbst Teil der Regierung sein kann. Das Kriterium drängt also nach weiterer Differenzierung, je nachdem, wie die Gewichte zwischen Präsident und Premier innerhalb der Exekutive verteilt sind. Liegt die Prärogative beim gewählten Präsidenten, handelt es sich nach Ansicht der meisten Autoren um einen Mischtyp zwischen Parlamentarismus und Präsidentialismus, für den sich der Begriff des »semi-präsidentiellen« Systems eingebürgert hat. ${ }^{5}$ Andere gehen bei der Aufschlüsselung noch weiter. So unterscheiden Shugart und Carey ${ }^{6}$ in ihrer häufig zitierten Darstellung zwei, Shugart ${ }^{7}$ in einer späteren Veröffentlichung sogar drei Varianten des semi-präsidentiellen Systems, die in verwirrender Begriffsvielfalt als "premier-präsidentiell«, "präsidentiell-parlamentarisch« oder »parlamentarisch mit Präsident « bezeichnet werden. ${ }^{8}$

3 Giovanni Sartori, Comparative Constitutional Engineering. An Inquiry into Structures, Incentives and Outcomes, 2. Auflage, Houndmills u.a. 1997, S. 85f.

4 Winfried Steffani, »Ministerpräsident und / oder Premierminister? « in: Tobias Dürr / Franz Walter (Hg.), Solidargemeinschaft und fragmentierte Gesellschaft, Opladen 1999, S. 235. 
Die Willkürlichkeit, die solchen und ähnlichen ad hoc-Konstruktionen anhaftet, ist evident. Sie lässt sich überwinden, wenn man dem methodisch stringenteren Verfahren der Typenbildung folgt, das die - überwiegend von deutschen Politikwissenschaftlern repräsentierte - zweite Schule vertritt. ${ }^{9}$ Die bis heute schlüssigste Klassifikation der parlamentarischen und präsidentiellen Regierungssysteme stammt von Winfried Steffani, dessen Arbeiten im angelsächsischen Sprachraum freilich kaum rezipiert worden sind. Steffani geht davon aus, dass für die Unterscheidung zwischen beiden Grundtypen letztlich ein singuläres Kriterium genügt, nämlich die Abberufbarkeit oder Nicht-Abberufbarkeit der Regierung. Die darauf fußende Typologie hat zum einen den Vorzug, dass sie die Existenz von Mischsystemen logisch ausschließt. Selbst ein System wie das schweizerische, dessen parlamentarische oder präsidentielle Qualität sich dem Beobachter nicht unmittelbar erschließt, kann damit eindeutig zugewiesen werden. (Es gehört zum präsidentiellen Typus.) Zum anderen erlaubt sie die Bildung von Untertypen, die anhand von formalen (Republik / Monarchie) oder materiellen Merkmalen (Machtverteilung) bestimmt werden. So unterscheidet Steffani unter den parlamentarischen Systemen solche mit Versammlungs-, Kabinetts-, Premierminister- oder Präsidialdominanz. Der letztgenannte Typus deckt sich in der Beschreibung nahezu vollständig mit dem Semi-Präsidentialismus à la Duverger, wird aber von Steffani eben nicht als ein eigenständiger Grundtyp betrachtet; es handelt sich gewissermaßen um ein »unechtes « Mischsystem. ${ }^{10}$

Ist das Zuordnungsproblem des »Semi-Präsidentialismus « damit gelöst, so schließt das nicht aus, dass es in einer erweiterten theoretischen Perspektive auch »echte« Mischsysteme geben könnte. Die Frage lautet, ob das Kriterium der Abberufbarkeit / Nicht-Abberufbarkeit zur Unterscheidung der beiden Grundtypen tatsächlich genügt. Die meisten Autoren halten zumindest ein weiteres Merkmal für

5 Vgl. u.a. Maurice Duverger, »A New Political System Model: Semi-Presidential Government « in: European Journal of Political Research 8 (1980), S. 165-187; Arend Lijphart, Democracies. Patterns of Majoritarian and Consensus Government in Twenty-One Countries, New Haven/London 1984; Horst Bahro / Ernst Veser, »Das semipräsidentielle System - „Bastard“ oder Regierungsform sui generis « in: Zeitschrift für Parlamentsfragen 26 (1995) H.3, S. 471-485; Bernhard Thibaut, »Präsidentielle, parlamentarische oder hybride Regierungssysteme? Institutionen und Demokratieentwicklung in der Dritten Welt und in den Transformationsstaaten Osteuropas « in: Zeitschrift für Politikwissenschaft 8 (1998) H.1, S. 5-37.

6 Matthew S. Shugart / John M. Carey, Presidents and Assemblies. Constitutional Design and Electoral Dynamics, Cambridge/New York 1992.

7 Matthew S. Shugart, »Of Presidents and Parliaments « in: East European Constitutional Review 2 (1993) H.1, S. 30-32.

8 Zur Kritik vgl. Jerzy Maćków, Parlamentarische Demokratie und Autoritarismus. Erfolge und Misserfolge der postkommunistischen Verfassunggebung, Hamburg 1998.

9 Vgl. z.B. Werner Kaltefleiter, Die Funktionen des Staatsoberhauptes in der parlamentarischen Demokratie, Köln/Opladen 1970; Winfried Steffani, Parlamentarische und präsidentielle Demokratie. Strukturelle Aspekte westlicher Demokratien, Opladen 1979.

10 Vgl. Winfried Steffani, "Semi-Präsidentialismus: ein eigenständiger Systemtyp? Zur Unterscheidung von Legislative und Parlament « in: Zeitschrift für Parlamentsfragen 26 (1995) H.4, S. 621-641. 
unabdingbar, das über die Bestellung (selection) der Regierung bzw. des Regierungschefs Auskunft gibt; diese erfolgt im parlamentarischen System stets durch das Parlament, während im präsidentiellen System der »chief executive« in direkter oder indirekter Wahl vom Volk eingesetzt wird. ${ }^{11}$ Steffani sieht demgegenüber das Kriterium der Bestellung in jenem der Abberufbarkeit bereits mit enthalten. Dahinter steht die Beobachtung, dass auch in den parlamentarischen Systemen der Regierungschef normalerweise nicht direkt durch das Parlament ins Amt gelangt, sondern vom monarchischen oder republikanischen Staatsoberhaupt ernannt wird. ${ }^{12}$ Dessen Entscheidungsspielraum ist jedoch durch das parlamentarische Abberufungsrecht weitgehend beschnitten, das der ernennenden Instanz anzeigt, wen (und wen nicht) sie in das Amt des Regierungschefs berufen darf. »Insofern hat das Institut des Misstrauensvotums gerade dank der Tatsache, dass es fast nie angewandt wird und wenn, dann zur Parlamentsauflösung führt, die positive politische Wirkung, dass der Parlamentswille bei der Regierungsbestellung allein ausschlaggebend bleibt « ${ }^{13}$.

Weniger Sinn macht die Reduktion auf das Kriterium der Abberufbarkeit / NichtAbberufbarkeit bei den präsidentiellen Systemen. In Steffanis Typologie führt dies dazu, dass auch ein nicht-demokratisches System wie die konstitutionelle Monarchie des Kaiserreiches zum Grundtyp des Präsidentialismus gerechnet wird. ${ }^{14}$ Ein präsidentielles System, das demokratischen Anforderungen genügt, kommt hingegen um das zusätzliche Erfordernis der Volkswahl nicht herum, wenn es die Nicht-Abberufbarkeit des Regierungschefs legitimatorisch ausgleichen will. Ob daraus eine im Vergleich zum parlamentarischen System höherwertige demokratische Qualität der Regierung resultiert, ist eine andere Frage. Die gewaltentrennende Logik des Präsidentialismus spricht nach Ansicht von Juan Linz eher dagegen, weil sie die Zurechnung politischer Verantwortung (die ein Wesensmerkmal der Demokratie ist) prinzipiell erschwert. Während die Verschränkung von Parlamentsmehrheit und Regierung im parlamentarischen System für klare Fronten sorgt, bleibt der chief executive im präsidentiellen System auf das Wohlwollen der Legislative angewiesen, hinter deren Rücken er sich notfalls verstecken kann. Zusätzlich verschärft wird das Problem durch die konstitutionelle Vorkehrung der Amtszeitbegrenzung, die (in den meisten Fällen) nur eine einmalige Wiederwahlmöglichkeit vorsieht: »[T]here is no way to hold accountable a president who cannot be presented for reelection. Such

11 Vgl. Leon D. Epstein, »Parliamentary Government« in: David L. Sills (Hg.), International Encyclopedia of the Social Sciences. Volume 11, New York/London 1968, S. 419-426.

12 Die formelle Wahl des Regierungschefs durch das Parlament - wie in Deutschland und Spanien - bleibt in den parlamentarischen Systemen die Ausnahme. In Spanien ist sie ein Ausdruck der intensiven Rezeption des Bonner Grundgesetzes, die in der Verfassung von 1978 auch zur Übernahme des konstruktiven Misstrauensvotums führte. In Deutschland muss ihre Einführung vor dem Hintergrund der fatalen Rolle gesehen werden, die Hindenburg 1933 bei der (keineswegs zwingenden) Ernennung Hitlers zum Reichskanzler gespielt hat.

13 Winfried Steffani, »Regierungsmehrheit und Opposition« in: ders. (Hg.) unter Mitarbeit von Jens-Peter Gabriel, Regierungsmehrheit und Opposition in den Staaten der EG, Opladen 1991, S. 17.

14 Steffani, aaO. (FN 10), S. 638. 
a president can neither be punished by the voters by defeat nor rewarded for success by reelection with the same or larger vote than in the previous election « ${ }^{15}$.

Bildet die Volkswahl des Regierungschefs ein notwendiges Merkmal des Präsidentialismus, dann wird auch die Zuordnung der Schweiz zum präsidentiellen Typus fraglich. Die Schweizer Regierung, der siebenköpfige Bundesrat, ist zwar vom Parlament nicht abberufbar; sie muss jedoch von diesem in einer förmlichen Wahl bestellt werden. Insofern scheint es sich um einen echten Mischtyp zwischen Parlamentarismus und Präsidentialismus zu handeln, worauf auch die Konstruktion der Exekutive hindeutet: Der Vorsitzende des Bundesrates ist einerseits als gleichberechtigter Teil in die Kollegialregierung integriert; andererseits nimmt er - als Bundespräsident - die Funktionen eines Staatsoberhauptes wahr. Die Verbindung von parlamentarischer Bestellung und Nicht-Abberufbarkeit der Regierung ist ein weltweites Unikum. In der Schweiz funktioniert sie deshalb, weil die (partei)politische Zusammensetzung des Bundesrates bereits vorab feststeht und vom Parlament nicht ohne weiteres verändert werden kann. Auch in personeller Hinsicht bleiben die Wirkungen des parlamentarischen Bestellungsrechts begrenzt, da es in der Bundesversammlung übliche Praxis ist, die einmal gewählten Regierungsmitglieder in ihren Ämtern zu bestätigen. Die Einzigartigkeit des Schweizer Modells liegt darin, dass es die Kontinuität der All-Parteien-Regierung im Inputbereich durch ein weitreichendes System direkter Demokratie ergänzt. Der Umstand, dass auf der Repräsentationsbene ein Regierungswechsel von der Hand des Wählers nicht herbeigeführt werden kann, wird damit legitimatorisch aufgefangen. Als Typus bewegt sich das Schweizer System so gesehen zwischen allen Stühlen. Es ist weder parlamentarisch noch präsidentiell und kann daher allenfalls mit Verlegenheitsbegriffen umschrieben werden (Rats- oder Kollegialsystem, independent assembly etc.).

Der theoretisch interessantere Fall eines Mischsystems liegt in der umgekehrten Konstellation: Der Regierungschef wird vom Volk gewählt, bleibt aber trotzdem weiterhin vom Vertrauen des Parlaments abhängig. Anders als im Schweizer Fall werden hier die prägenden Merkmale der beiden Systemtypen tatsächlich kombiniert, handelt es sich also um eine wirkliche Synthese. Bis zur Verfassungsreform in Israel gab es für das "präsidentiell-parlamentarische "Modell allerdings kein einziges empirisches Beispiel, sodass die Typologie der Regierungssysteme in diesem Feld unbesetzt bleiben musste. ${ }^{16}$ Mit der 1992 beschlossenen und 1996 erstmals durchgeführten Direktwahl des israelischen Premierministers lässt sie sich nun vervollständigen. ${ }^{17}$

15 Juan J. Linz, »Presidential or Parliamentary Democracy: Does It Make a Difference? in: ders. / Arturo Valenzuela (Hg.), The Failure of Presidential Democracy, Baltimore/ London 1994, S. 12.

16 Lijphart, aaO. (FN 5), S. 70.

17 Die Typologie weicht von der Lijphart'schen Vorlage darin ab, dass sie bei der Bestellung nicht auf den »chief executive«, sondern den Regierungschef (Premierminister) Bezug nimmt. Dies hat den Vorteil, dass über die Frage, wer der wahre Chef der Exekutive ist, nicht vorab entschieden werden muss und vermeidet so die Zuordnung der semi-präsidentiellen Systeme zum Grundtyp des Präsidentialismus - ein Kardinalfehler in Lijpharts Typologie. Wird der Premierminister betrachtet, so liegt das Recht der Bestellung - wie oben gezeigt auch in den semi-präsidentiellen Systemen beim Parlament, weshalb diese zum Grundtyp des Parlamentarismus gehören. Dass der Präsident an der Bestellung der Regierung beteiligt ist und dabei ein womöglich entscheidendes Wort mitzureden hat, steht dem nicht entgegen. 
Abb. 1: Typologie parlamentarischer und präsidentieller Regierungssysteme

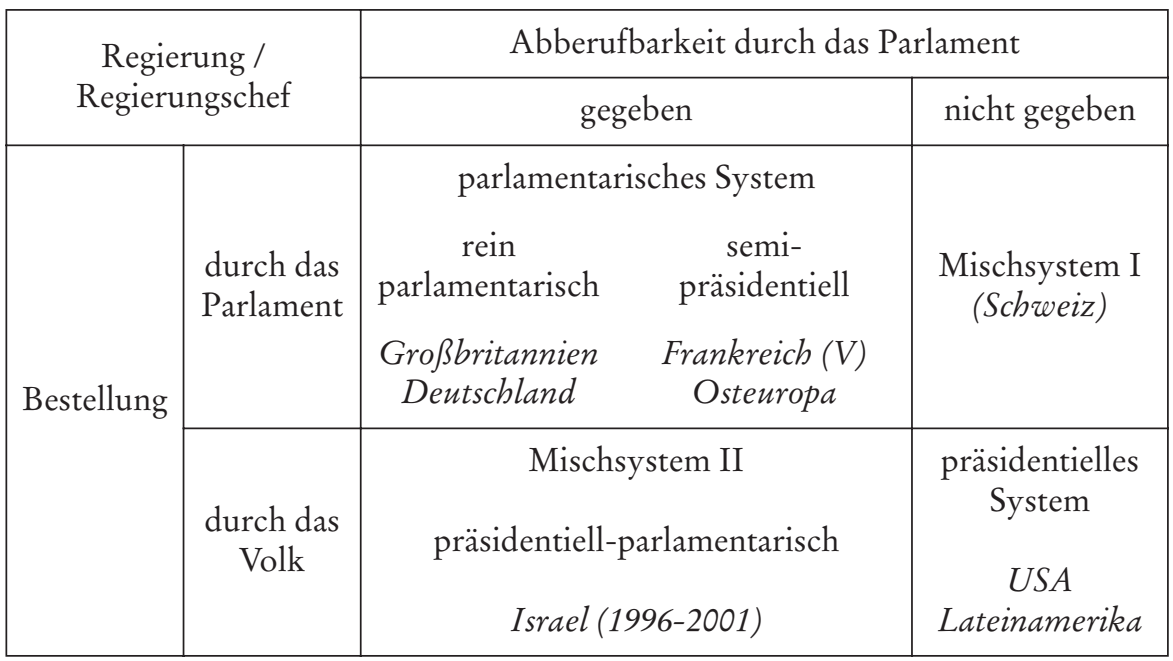

Der Vorschlag, den Premier unabhängig von der Wahl der Legislative in unmittelbarer Volkswahl zu bestellen, war erstmals Ende der sechziger Jahre von der neu gegründeten Partei Demokraten '66 für die Niederlande ins Gespräch gebracht, dort aber nicht weiter verfolgt worden. ${ }^{18}$ Erst die Krise der demokratischen Institutionen in Israel sollte ihm in den neunziger Jahren zum Durchbruch verhelfen. Von namhaften Politikwissenschaftlern des Landes mit ersonnen, stellte die israelische Verfassungsreform von 1992 den ausdrücklichen Versuch dar, die jeweiligen Vorzüge des parlamentarischen und präsidentiellen Systems miteinander zu verknüpfen. Das Experiment entpuppte sich als gewaltiger Fehlschlag, sodass die Direktwahl, die 1996 erstmals stattfand, schon nach fünf Jahren wieder abgeschafft wurde und Israel zu einer leicht modifizierten Variante des alten parlamentarischen Systems zurückkehrte. Die nachfolgenden Ausführungen gehen zunächst auf die Hintergründe des »präsidentiellen Parlamentarismus « ein und schildern die verschiedenen Etappen bis zur Verabschiedung des Reformgesetzes. Anschließend wird untersucht, wie sich das Direktwahlsystem ausgewirkt hat und warum sein Scheitern letztlich unabwendbar war.

18 Vgl. J.P.A. Gruijters, »The Case for a Directly Elected Prime Minister in the Netherlands" in: Arend Lijphart (Hg.), Parliamentary versus Presidential Government, Oxford 1992, S. 191-193. 


\section{Die Einführung des "präsidentiellen Parlamentarismus« in Israel}

\section{a) Vorgeschichte}

Das politische System Israels stellt für die Vergleichende Politikwissenschaft eine echte Herausforderung dar. Die Neuerrichtung einer nationalen Heimstätte, die 2000 Jahre von der Landkarte verschwunden war, das Selbstverständnis als jüdischer Staat und seine exponierte Sicherheitslage machen Israel unter den demokratischen Ländern zu einem »unvergleichlichen Fall. $^{19}$ Bis heute ist Israel die einzige verfassungsstaatliche Demokratie des Nahen Ostens geblieben. An diese Tatsache muss deshalb erinnert werden, weil von den kulturellen, wirtschaftlichen und politischen Begleitumständen her der Weg dahin keineswegs vorgezeichnet war. Weder geografisch noch politisch-kulturell handelt es sich bei Israel um ein »westliches« Land. Dagegen spricht nicht nur der Umstand, dass die orientalischen Sephardim inzwischen die Mehrheit der jüdischen Bevölkerung bilden - zum Zeitpunkt der Staatsgründung 1948 war sie noch zu mehr als vier Fünfteln aschkenasischer (= europäisch-amerikanischer) Herkunft. ${ }^{20}$ Auch die Pioniere der Gründergeneration, die wie der erste Premierminister David Ben-Gurion überwiegend aus Osteuropa und Russland stammten, waren in ihrer politischen Vorstellungswelt eher von sozialistischen als liberalen Ideen geprägt worden, was sie allerdings nicht daran hinderte, die » prozeduralen « Bedingungen der Demokratie zu respektieren. ${ }^{21}$

Das Überleben der Demokratie grenzt fast an ein Wunder, wenn man an die schweren Belastungen denkt, denen die israelische Gesellschaft in der Folgezeit ausgesetzt gewesen ist. Die äußere Sicherheitslage hat sich zwar mittlerweile entschärft, nachdem das Land mit zwei seiner ehemaligen Erzfeinde - Ägypten und Jordanien - Frieden schließen konnte. Ein entsprechendes Abkommen mit dem syrischen Nachbarn steht aber (auch nach dem Rückzug der israelischen Armee aus dem Südlibanon) weiterhin aus. Ungleich schwerer noch als die äußeren Gefahren wiegen die inneren Probleme, die sich Israel mit seinem militärischen Sieg im Sechstagekrieg (1967) eingehandelt hat. Die bis heute ungelöste Palästinenserfrage wirft auf das demokratische Selbstverständnis des Landes einen dunklen Schatten. Der Status einer Besatzungsmacht rührt ja nicht nur an die legitimen Besitzrechte des palästinensischen Volkes; er macht auch einen unbefangenen Umgang mit der im Kernland verbliebenen arabisch-palästinensischen Minderheit unmöglich, die immerhin ein Fünftel der israelischen Gesamtbevölkerung umfasst. Letzteres verweist auf das unter demokratischen Gesichtspunkten prekäre Problem der jüdischen Identität Israels, die einem aufgeklärten (ethnisch neutralen) Staats- und Nationverständnis im

19 Vgl. Benyamin Neuberger, "Israel's Democracy and Comparative Politics « in: Jewish Political Studies Review 1 (1989) H.3-4, S. 67-75.

20 Vgl. Michael Wolffsohn / Douglas Bokovoy, Israel. Geschichte, Politik, Gesellschaft, Wirtschaft (1882 - 1994), 4. Auflage, Opladen 1995, S. $257 \mathrm{ff}$.

21 Vgl. Jonathan Shapiro, "The Historical Origins of Israeli Democracy« in: Ehud Sprinzak / Larry Diamond (Hg.), Israeli Democracy under Stress, Boulder/London 1993, S. 65-79. 
Wege steht, für den Zusammenhalt der nach Israel eingewanderten Juden aber weiterhin eine unverzichtbare Quelle darstellt. ${ }^{22}$

Gefahren drohen der israelischen Demokratie heute primär durch die wachsende innerjüdische Polarisierung. Wirkten die äußere Bedrohung und das Aufbauwerk im Inneren bis zum Sechstagekrieg solidaritätsstiftend, so zeigt sich das Land seither über der Frage nach dem angemessenen Umgang mit den besetzten Gebieten tief zerstritten. Die besondere Brisanz des Konfliktes rührt daher, dass er durch die vorhandenen Spannungslinien der israelischen Gesellschaft aufgeladen und verstärkt wird. Neben der erwähnten ethnischen Spaltung der Bevölkerung in Aschkenasim und Sephardim betrifft das vor allem den Konflikt zwischen säkularen und strenggläubigen Juden, der auf das Kernproblem des jüdischen Charakters Israels zurück verweist. ${ }^{23}$ Obwohl die Orthodoxen nur etwa zehn Prozent der israelischen Juden ausmachen (in Jerusalem liegt ihr Anteil bei rund einem Viertel), kommt der Religion in Staat und Politik traditionell große Bedeutung zu. Die Gründe dafür sind zunächst institutioneller Natur. Um den inneren Frieden nicht zu gefährden, hatten die überwiegend säkular eingestellten zionistischen Staatsgründer nach 1948 wesentliche Bereiche des öffentlichen Lebens in der Verantwortung der religiösen Instanzen belassen (und dabei zugleich den Monopolanspruch der orthodoxen Juden festgeschrieben). Aus demselben Grund verzichtete man bewusst darauf, dem Staat eine schriftliche Verfassung zu geben, und begnügte sich stattdessen mit mehreren sogenannten »Grundgesetzen «, die den Aufbau der zentralen Staatsorgane regeln sollten. ${ }^{24}$ Erst in jüngster Zeit zeichnen sich Ansätze einer stärkeren verfassungsgerichtlichen Kontrolle ab, deren Ausbau zu einem vollgültigen Grundrechtssystem von den religiösen Parteien aber nach wie vor blockiert wird. ${ }^{25}$

Ist ihre institutionelle Stellung damit abgesichert, so muss der Aufstieg der religiösen Kräfte zu einem relevanten Machtfaktor vor dem Hintergrund der politischen und gesellschaftlichen Veränderungen gesehen werden, die Israel seit dem Sechstagekrieg durchlaufen hat. Den ersten Hinweis liefert auch hier die Demografie. Die Masseneinwanderung der orientalischen Juden konnte zwar den allgemeinen Säkularisierungsprozess im Lande nicht stoppen; sie führte jedoch dazu, dass sich innerhalb des religiösen Lagers die Gewichte zugunsten der radikaleren, nicht-zionistischen Vertreter verschoben, die heute vor allem von der Schas-Partei repräsentiert werden. ${ }^{26}$ In der konsensuell geprägten Atmosphäre bis zum Sechstagekrieg hatte die Einbeziehung der überwiegend pro-zionistisch ausgerichteten orthodoxen Parteien in die Regierungspolitik wenig Probleme bereitet, da das Parteiensystem klar

22 Vgl. Ira Sharkansky, Policy Making in Israel. Routines for Simple Problems and Coping with the Complex, Pittsburgh 1997, S. 71ff.

23 Vgl. Asher Arian, The Second Republic. Politics in Israel, Chatham 1998, S. 6ff.

24 Reuven Y. Hazan, »Executive-Legislative Relations in an Era of Accelerated Reform: Reshaping Government in Israel in: Legislative Studies Quarterly 22 (1997) H.3, S. $329 f$.

25 Vgl. Larry Diamond / Ehud Sprinzak, »Directions for Reform« in: dies., aaO. (FN 21), S. $369 \mathrm{ff}$.

26 Vgl. Arian, aaO. (FN 23), S. 127ff. 
von der Arbeitspartei (Mapai) beherrscht wurde. Nach 1967 veränderte sich die Lage von Grund auf. Über den Streit um das Besatzungsregime kam es jetzt zu einer stärkeren Politisierung, die das religiöse Lager von der führenden Regierungspartei allmählich entfremdete. Die Annäherung der orthodoxen Vertreter an die erstarkenden rechtsnationalen Kräfte mündete in den historischen Machtwechsel von 1977, der die Ära des »dominanten « Parteiensystems in Israel beendete. ${ }^{27}$ Die religiösen Parteien gerieten fortan immer mehr in die strategisch vorteilhafte Situation eines »Königsmachers «, dessen Unterstützung darüber entscheidet, welcher der beiden großen Blöcke die Regierung bilden kann. Ihr politischer Einfluss vergrößerte sich damit zusehends und begann, über die tatsächliche elektorale Stärke hinauszureichen.

Nun ist es in parlamentarischen Mehrparteiensystemen nichts Ungewöhnliches, dass kleinere Parteien bei der Regierungsbildung eine Züngleinrolle spielen und dadurch das demokratische Wählervotum verzerren können. Dies gilt zumal dann, wenn die Wähler über die gewünschten Koalitionspräferenzen von den Politikern vorab im Unklaren gelassen werden. In Israel haben die Verzerrungen freilich ein groteskes Ausmaß erreicht und mit dazu beigetragen, dass die seit den achtziger Jahren immer häufiger auftretenden Regierungskrisen in eine permanente Systemkrise umgeschlagen sind. Ursächlich dafür war eine Kombination besonderer Problemumstände:

- Erstens leistete das aus der vorstaatlichen Zeit übernommene Verhältniswahlrecht einer extremen Fragmentierung der Parteienlandschaft Vorschub, die innerhalb des religiösen Lagers zu zahlreichen Abspaltungen und Neugründungen führte. ${ }^{28}$ Weil die Zugangsschwellen zum Parteiensystem sehr niedrig waren - die Sperrklausel lag bei lediglich einem Prozent -, gab es für die einzelnen Gruppierungen keinen Anreiz, sich zusammenzuschließen oder auf ein gemeinsames politisches Programm zu einigen. Die führende Regierungspartei hatte es daher stets mit einem halben Dutzend oder mehr Koalitionspartnern zu tun, von denen jeder einzelne für die Mehrheitsbildung ausschlaggebend sein konnte.

- Zweitens profitierten die religiösen Parteien von der Struktur der innenpolitischen Agenda in Israel, die durch eine klare Priorität der sicherheits- und friedenspolitischen Themen geprägt ist. Dies begünstigte eine spezielle Form der Arbeitsteilung, bei der sich die Koalitionspartner auf ihren jeweiligen Interessengebieten mehr oder weniger freie Hand ließen. Während die religiösen Vertreter das sicherheitspolitische Vorgehen der führenden Regierungspartei mittrugen, verpflichtete sich diese im Gegenzug, die Privilegien der religiösen Institutionen im öffentlichen Bereich nicht anzutasten. Die orthodoxen Parteien kamen dadurch in den Genuss weitreichender Zugeständnisse, die insbesondere das Eherecht und Erziehungswesen betrafen.

27 Vgl. Gershon R. Kieval / Bernard Reich, »Introduction: The Changing Center in Israeli Politics « in: dies. (Hg.), Israeli Politics in the 1990s, Newport/Westport/London 1991, S. 1-27.

28 Vgl. Wolffsohn / Bokovoy, aaO. (FN 20), S. $138 f f$. 
- Drittens schließlich muss die Rivalität der beiden großen Blöcke berücksichtigt werden. Arbeitspartei und Likud hatten sich seit den achtziger Jahren in ihren friedenspolitischen Vorstellungen so weit voneinander entfernt, dass sie für eine Regierung unter Ausschluss des anderen einen hohen Preis zu zahlen bereit waren. Wollte man den Stillstand einer Großen Koalition vermeiden, gab es zu einer Einbeziehung der religiösen Parteien also keine Alternative. Das elektorale Patt zwischen »Tauben « und »Falken « und ihre eigene ideologische Offenheit nach beiden Seiten brachten diese in eine Position, in der sie den Kompromisswillen der größeren Regierungspartei bis aufs äußerste strapazieren konnten. ${ }^{29}$

Im Frühjahr des Jahres 1990 erreichte die Krise einen traurigen Höhepunkt, der die Reformnotwendigkeit der demokratischen Institutionen für jedermann offensichtlich machte. Nachdem es bei den vorangegangenen Parlamentswahlen (1984 und 1988) weder der Arbeitspartei noch dem Likud gelungen war, eine eigene Regierungsmehrheit zustande zu bringen, hatten sich beide Parteien 1984 widerwillig in eine Große Koalition gefügt. Um das Zusammengehen überhaupt zu ermöglichen, entwickelten sie dabei einige institutionelle Phantasie. So verständigte man sich z.B. darauf, das Amt des Premierministers rotieren zu lassen, wofür es in den westlichen Demokratien bis dahin kein Vorbild gegeben hatte..$^{30}$ Nach anfänglich durchaus vorzeigbaren Resultaten (u.a. bei der Inflationsbekämpfung) machten die unüberbrückbaren Differenzen in der Palästinenserfrage den Bruch spätestens seit Ausbruch der Intifada unabwendbar. Anfang 1990 kündigte die von Außenminister Schimon Peres angeführte Arbeitspartei die Zusammenarbeit auf und brachte die Regierung anschließend mit dem ersten erfolgreichen Misstrauensvotum in der noch jungen Geschichte des Staates zu Fall. Peres' Bemühungen, eine neue Mehrheit unter Ausschluss des Likud zu bilden, blieben allerdings ergebnislos ${ }^{31}$ und endeten in einem absurden Geschacher, bei dem sich die großen Parteien in ihrem Buhlen um Unterstützung wechselseitig überboten. »Hundreds of millions of government dollars were readily committed as coalition bribery to tiny parties. Top ministerial and bureaucratic positions were offered to inexperienced and corrupt MKs [Knessetabgeordnete, FD] in exchange for their votes. Several especially un-

29 Arian, aaO. (FN 23), S. 129.

30 Auch nachher hat man eine solche Lösung nirgendwo praktiziert. Kurzzeitige Prominenz erlangte das Modell anlässlich der Bundestagswahl 2005 in Deutschland, als die Rotation von der SPD als Kompromissformel ins Spiel gebracht wurde, um die Kanzlerschaft Gerhard Schröders im Rahmen der neu zu bildenden Großen Koalition zu verlängern. Dies erwies sich allerdings gegen die CDU / CSU als nicht durchsetzbar, die bei der Wahl knapp vor der SPD gelegen hatte und damit auf den gewohnheitsrechtlichen Anspruch pochen konnte, wonach der stärkere Partner in einer Koalition das Amt des Regierungschefs besetzt.

31 Peres begründete damit seinen inzwischen legendären Ruf als geborener Wahlverlierer, der ihn später auch gegen Jitzchak Rabin (im innerparteilichen Vorwahlduell 1992), Benjamin Netanjahu (bei der Direktwahl des Premierministers 1996), Mosche Katzav (bei der Wahl des Staatspräsidenten 2000) und Amir Peretz (im Duell um den Parteivorsitz 2005) unterliegen ließ. 
scrupulous MKs used the opportunity to split from their mother parties, instantly tripling and quadrupling their price in the political supermarket « ${ }^{32}$.

\section{b) Von der Wablrechtsreform zum Direktwablvorschlag}

Auch wenn am Ende eine neue, vom Likud geführte Regierung mit Jitzchak Schamir an der Spitze gebildet werden konnte, lag doch das eigentlich Positive der Krise darin, dass sie die Reform des Systems nun offiziell auf die politische Tagesordnung setzte. Am nächsten lag dabei natürlich eine Änderung des Wahlrechts, das in Israel von Beginn an umstritten gewesen war. Die Wahlrechtsreform verfolgte zwei miteinander verbundene Ziele: Einerseits sollte sie die Zahl der im Parlament vertretenen Parteien reduzieren und dadurch die Regierungsbildung (sowie das Regieren selbst) erleichtern, andererseits die demokratischen Einflussmöglichkeiten des Wählers verbessern. Letztere sind in dem bestehenden Verhältniswahlsystem aufgrund der fehlenden Wahlkreiseinteilung begrenzt, sodass die Wähler bislang nur zwischen nationalen Parteilisten - statt individuellen Wahlkreiskandidaten - auswählen konnten. ${ }^{33}$

Es ist eine politologische Binsenweisheit, dass Wahlsysteme, wenn sie einmal etabliert sind und den gesellschaftlichen Verhältnissen Rechnung tragen, ein hohes Beharrungsvermögen besitzen. Die Liste der gescheiterten Reformen in Israel bestätigt diese These eindrucksvoll. Sie beginnt bereits in den fünfziger Jahren, als die Mapai mehrere vergebliche Anläufe unternahm, das Verhältniswahlrecht durch ein Mehrheitswahlsystem nach britischem Muster zu ersetzen. Die Versuche riefen den gemeinsamen Widerstand der kleineren Parteien hervor, denen es in der Folge gelang, die verfassungsrechtlichen Hürden für eine Wahlrechtsreform zu erhöhen. Die Funktionsmängel des Wahlsystems, die durch die hegemoniale Position der Arbeitspartei lange Zeit überdeckt worden waren, ließen sich spätestens nach dem Übergang zur Bipolarität (ab 1977) nicht mehr verbergen. Arbeitspartei und Likud hätten jetzt zwar eine Reform gegen den Willen der anderen Parteien theoretisch beschließen können, doch hinderte sie daran ihr wechselseitiges Misstrauen. Beide Seiten standen in der Wahlrechtsfrage vor einer typischen "Prisoner's dilemma «-Situation, die es ihnen nahe legte, eine Nicht-Einigung um des kurzfristigen Machtvorteils willen zu bevorzugen. ${ }^{34}$ Selbst der moderate Reformvorschlag der YaacobiKommission, der eine personalisierte Form des Verhältniswahlrechts anstrebte ${ }^{35}$,

32 Ehud Sprinzak / Larry Diamond, »Introduction« in: dies., aaO. (FN 21), S. 4.

33 Vernon Bogdanor, »The Electoral System, Government and Democracy « in: Sprinzak / Diamond, aaO. (FN 21), S. 85.

34 Hannah Diskin / Abraham Diskin, »The Politics of Electoral Reform in Israel in: International Political Science Review 16 (1995) H.1, S. 36.

35 Die Hälfte der 120 Knessetabgeordneten sollte danach in 20 Dreierwahlkreisen mit einfacher Mehrheit, die andere Hälfte wie bisher per Listenwahl gewählt werden, wobei sich das Stimmenverhältnis ausschließlich nach dem Ergebnis der Listenwahl bemessen hätte. Als Sperrklausel war ein Minimum von vier gewonnenen Mandaten vorgesehen, was einer Quote von 3,3 Prozent entspricht. Vgl. Avraham Brichta, „Choosing an Electoral System « in: The Jewish Journal of Sociology 33 (1991), S. 89ff. 
hatte unter diesen Bedingungen keine Verwirklichungschance und gelangte 1990 nicht einmal mehr in das parlamentarische Beratungsstadium. ${ }^{36}$ Als einziges greifbares Ergebnis konnte 1992 eine leichte Anhebung der Sperrklausel (auf 1,5 Prozent) durchgesetzt werden, die freilich nicht ausreichte, um das beschriebene Problem in den Griff zu bekommen: Nachdem die Zahl der im Parlament vertretenen Parteien von 15 (1984 und 1988) auf 10 (1992) bzw. 11 (1996) vorübergehend sank, erreichte sie bereits 1999 wieder den alten Stand.

Das Scheitern der Wahlrechtsreform führte dazu, dass sich die Debatte seit Ende der achtziger Jahre zunehmend auf ein anderes Feld verlagerte: das der exekutiv-legislativen Beziehungen. Auch hier ging es um die doppelte Frage, wie das Erpressungspotenzial der kleineren Parteien reduziert und der Einfluss der Wähler auf die Regierungsbildung verstärkt werden könnte. Über die grundsätzliche Lösung des Problems herrschte unter den Reformern Einigkeit: Sie bestand darin, die Position des Premierministers innerhalb der Regierung aufzuwerten. Unklar war jedoch, wie dieses Ziel am besten erreicht werden konnte. Im Prinzip boten sich dafür zwei Wege an: Der erste Weg orientierte sich am Westminster-Modell des »Prime Ministerial Government « und verband dies mit der Einführung eines »konstruktiven Misstrauensvotums « nach bundesdeutschem Vorbild. ${ }^{37}$ Der zweite Weg führte auf verfassungspolitisches Neuland; er sah vor, den Premierminister künftig direkt vom Volk wählen zu lassen.

Dass die Reform am Ende tatsächlich verwirklicht wurde (ob sie auch zum Erfolg führte, ist eine andere Frage, die weiter unten erörtert werden soll), verweist auf die im Vergleich zur Wahlrechtsänderung "günstigere « Interessenlage der betroffenen Akteure. Da eine institutionelle Aufwertung des Premierministers die Position der als Koalitionspartner benötigten kleineren Parteien nicht unmittelbar bedrohte, hatten Arbeitspartei und Likud diesmal keinen Grund, sich einer Lösung zu verweigern. Schwieriger zu beantworten ist, warum von den beiden Alternativen ausgerechnet der radikalere Direktwahlvorschlag zum Zuge kam, von dem die Reformer wussten, dass er den Charakter des bisherigen Regierungssystems nachhaltig verändern würde. Juan Linz ${ }^{38}$ hat darauf hingewiesen, dass Systeme mit einer starken Exekutive vorzugsweise in Krisen- oder Umbruchzeiten »installiert« werden, wenn der Ruf nach einer ordnenden Hand laut wird. Die von ihm als Beispiele genannten »semi-präsidentiellen « Verfassungen Frankreichs (V. Republik) und Portugals lassen sich auf den israelischen Fall allerdings kaum übertragen, weil sie mit der Einführung eines ganz neuen Systemtypus verbunden waren. (Dasselbe gilt für die semi-päsidentiellen Systeme, die nach dem Zusammenbruch des Kommunismus in Osteuropa errichtet wurden.) In Israel stand eine Totalrevision demgegenüber niemals zur Debatte. Der »unfertige« Zustand der Verfassung ermöglichte es hier, die

36 Philipp A. Keller, »Die Direktwahl des Premierministers. Israels Verfassungsreform von 1992 in: Zeitschrift für Politikwissenschaft 8 (1998) H.2, S. 603.

37 Vgl. Gideon Doron, »Electoral Reforms in a Veil of Uncertainty: The Case of Israel « in: International Problems / Society and Politics 55 (1990) H.3-4, S. 48ff.; Diskin / Diskin, aaO. (FN 34), S. 39f.; Keller, aaO. (FN 36), S. 608f.

38 Linz, aaO. (FN 15), S. 50. 
Reform von innen heraus anzugehen, ohne die Grundstruktur des vorhandenen Systems zu zerstören. Die Attraktivität des Direktwahlvorschlags bestand so gesehen in dem günstigen Verhältnis von Aufwand und Ertrag; er erforderte relativ wenig formale Eingriffe, die aber de facto auf einen Systemwechsel (vom reinen zum präsidentiellen Parlamentarismus) hinausliefen.

Eine zweite Erklärung muss bei der Genese des Direktwahlvorschlags ansetzen. 1984 in einem Zeitungsartikel erstmals aufgetaucht, war dieser bereits 1986 von einer Expertengruppe in ein sorgfältig ausgearbeitetes Reformkonzept umgegossen worden und dadurch früher an die Öffentlichkeit gelangt als der alternative Plan des »Prime Ministerial Government « ${ }^{39}$. Das Konzept entwickelte in der Folge seine eigene Dynamik. Dass die Direktwahl in der Bevölkerung breite Unterstützung hervorrufen würde, verstand sich ohnehin fast von selbst, versprach sie doch aus der Sicht der Wähler »mehr Demokratie«. Letztlich ausschlaggebend waren aber auch hier die Ereignisse von 1990. Unter ihrem Eindruck nahm die Zahl der Befürworter so stark zu, dass sich die Politiker ein Scheitern der Reform nicht mehr leisten konnten; jetzt es ging nur noch darum, in welcher Form die Direktwahl eingeführt werden würde. ${ }^{40}$

Damit wendet sich der Blick zu dem wohl wichtigsten Erklärungsfaktor: den Interessen der politischen Akteure. Hier wirkte es sich zunächst positiv aus, dass die Direktwahl etwas völlig Neuartiges darstellte, für das man auf ausländische Vorbilder nur bedingt zurückgreifen konnte. So sehr sich die Reformer über die allgemeine Tragweite ihres Vorhabens im Klaren waren, so wenig Vorstellungen hatten sie davon, welche genauen Auswirkungen die Direktwahl auf das politische System und seine Akteure haben würde. Dies bedeutet natürlich nicht, dass es die Beteiligten dem Zufall überlassen hätten, ob sie am Ende zu den Gewinnern oder Verlieren der Reform gehörten. Ihre Zustimmung war nur zu erlangen, wenn sich das mit der Direktwahl verbundene Risiko eines eigenen Machtverlustes in Grenzen hielt. Gerade diese Erwartung wurde durch das Vorhaben freilich genährt, das eine Stärkung der Autorität des Premierministers versprach, ohne den parlamentarischen Charakter der Verfassung zu beseitigen. Von daher brauchten weder das Parlament als ganzes noch die einzelnen Abgeordneten zu fürchten, in dem neuen System auf der Strecke zu bleiben. ${ }^{41}$

\section{c) Der ursprüngliche Entwurf des Reichman-Komitees}

Traf der Direktwahlvorschlag im allgemeinen auf günstige Umstände, so war seine Verwirklichung doch alles andere als ein Selbstgänger. Dass eine Reform von diesem Ausmaß eine ausführliche parlamentarische Beratung erfordern würde, lag auf der Hand. Von der Vorlage des ersten Entwurfs bis zur endgültigen Verabschiedung des Gesetzes im März 1992 vergingen schließlich sechs Jahre, in deren Verlauf das

39 Vgl. Keller, aaO. (FN 36), S. 602ff.

40 Diskin / Diskin, aaO. (FN 34), S. $40 f$.

41 Hazan, aaO. (FN 24), S. 344f. 
ursprüngliche Konzept an mehreren Stellen verändert - Kritiker würden sagen: verwässert - wurde.

Die Expertengruppe, die den ersten Entwurf ausgearbeitet hatte, setzte sich aus sieben Rechts- und Politikwissenschaftlern der Universität Tel Aviv zusammen, von denen einer - Amnon Rubinstein - zugleich Abgeordneter der Knesset war. Durch die Bildung eines öffentlichen Komitees (unter dem Vorsitz von Uriel Reichman) sorgte die Gruppe dafür, dass ihre Reformvorstellungen über die akademischen Kreise hinaus der Öffentlichkeit und den Politikern bekannt gemacht wurden. Dabei kam ihr zugute, dass das Direktwahlkonzept gut durchdacht war und auch mögliche Gegenargumente berücksichtigte. ${ }^{42}$

Die Autoren bezeichneten das von ihnen angestrebte Regierungssystem in einem ebenso hässlichen wie treffenden Kunstwort als "parliadential«. Damit wollten sie betonen, dass das System eine Mischung zwischen »Parlamentarismus « und »Präsidentialismus « darstellte, deren positive Elemente zu einer neuartigen und besseren Regierungsform vereinigt werden sollten. ${ }^{43}$ Der präsidentielle Charakter dieser Mischung kam in der vorgesehenen Direktwahl zum Ausdruck, die den Premierminister mit dem Parlament legitimatorisch auf eine Stufe stellen würde. Der parlamentarischen Logik entsprach, dass derselbe Premierminister mitsamt seiner Regierung weiterhin vom Vertrauen des Parlaments abhing und von diesem nötigenfalls abberufen werden konnte. Die Einführung eines solchen Mischsystems eröffnete nach Ansicht des Reichman-Komitees einen Ausweg aus dem bisherigen Reformdilemma. Das Problem lag darin, dass die bestehende parlamentarische Regierungsform zunehmend krisenanfälliger wurde, ein rein präsidentielles System für Israel aber ebenso wenig in Betracht kam wie ein semi-präsidentielles System nach französischen Muster. Beide Alternativen hätten einen radikalen Umbau erfordert, für den die israelische Politik in ihrer Kombination von ideologischer Polarisierung, Mehrparteiensystem und Verhältniswahlrecht schlechte Voraussetzungen bot. ${ }^{44}$ Der Entwurf des Reichman-Komitees hatte demgegenüber den Vorzug, dass er auf der vorhandenen (parlamentarischen) Grundstruktur aufbaute. Die Tel Aviver Professoren hofften dadurch, der Gefahr eines Stillstandes (deadlocks) zwischen Regierung und Parlament begegnen zu können, die nach ihrer Auffassung das Hauptproblem der präsidentiellen und semi-präsidentiellen Systeme darstellte. ${ }^{45}$

Die Eckpunkte des neuen »präsidentiell-parlamentarischen « Systems lauteten in dem Entwurf des Reichman-Komitees wie folgt:

42 Yoram Peri (Hg.), Electoral Reform in Israel. A Report of the First Working Session of the IDI International Forum on Electoral Systems Held from March 28 - April 2 at the Dan Caesarea Hotel, Tel Aviv 1989 (Israel Diaspora Institute).

43 Bernard Susser, »,Parliadential Politics': A Proposed Constitution for Israel « in: Parliamentary Affairs 42 (1989) H.1, S. 113.

44 Vgl. Avraham Brichta / Yair Zalmanovitch, »The Proposals for Presidential Government in Israel. A Case Study in the Possibility of Institutional Transference« in: Comparative Politics 19 (1986), S. 57-68.

45 Susser, aaO. (FN 43), S. $121 \mathrm{f}$. 
1) Die Bestimmungen zur Direktwahl sahen vor, dass der Premierminister in einem zweistufigen Verfahren ermittelt wird. Im ersten Wahlgang sollte eine Mehrheit von 45 Prozent für die Wahl genügen, deren Erreichung durch einen Bewerber man freilich für unwahrscheinlich hielt. Von daher würde erst die erwartete Stichwahl zwischen den beiden Bestplatzierten zusammen mit den Parlamentswahlen stattfinden. Auf diese Weise wollte man sicherstellen, dass die Mehrheitsverhältnisse zwischen den beiden Organen nicht auseinander fielen.

2) Für die parlamentarische Abberufung des Premierministers schrieb der Entwurf eine qualifizierte Mehrheit von 60 Prozent +1 (entsprechend 73 von 120 Stimmen) vor, die in der Knesset bis dahin noch nie erreicht worden war. Erschwerend kam hinzu, dass bei einem erfolgreichen Misstrauensvotum gleichzeitige Neuwahlen des Parlaments und des Premiers stattzufinden hatten. Dasselbe galt für den Fall, dass der Premierminister von sich aus zurücktrat. Der Regierungschef verfügte also über das in parlamentarischen Systemen übliche Auflösungsrecht. Um ihre Gesetzesvorschläge durchzubringen, bedurfte die Regierung weiterhin einer parlamentarischen Mehrheit von 50 Prozent +1 (entsprechend 61 Stimmen).

3) Innerhalb der Regierung sollte der Premierminister seine Minister ernennen und entlassen dürfen und die Organisationsgewalt besitzen. Darüber hinaus wollte man durch ein striktes Inkompatibilitätsgebot verhindern, dass ein zurückgetretener oder entlassener Minister jederzeit auf seinen Abgeordnetensitz zurückkehren konnte. Auf der anderen Seite stand der Knesset das Recht zu, einem Minister innerhalb von 30 Tagen nach seiner Ernennung (mit einer qualifizierten Mehrheit von 60 Prozent +1$)$ das Misstrauen auszusprechen.

Zusammengefasst stellte der Entwurf eine Kombination aus drei Gruppen von Elementen dar. Die erste Gruppe bestand aus den präsidentiellen Elementen, die in das System neu eingeführt werden sollten. Dazu gehörten als Hauptmerkmal die Direktwahl des Regierungschefs sowie als weitere supplementäre Merkmale die Unvereinbarkeit von Regierungsamt und Abgeordnetenmandat und das parlamentarische Bestätigungsrecht der Minister. Die zweite Gruppe umfasste Elemente, die aus dem bestehenden parlamentarischen System in veränderter Form übernommen wurden. Dies galt zum einen für die Abberufbarkeit der Regierung (als Hauptmerkmal), zum anderen für das supplementäre Merkmal der Parlamentsauflösung. In beiden Fällen waren die Regelungen so ausgestaltet, dass sie die Position der Regierung gegenüber dem Parlament stärkten. Eine dritte Gruppe von supplementären Merkmalen des Parlamentarismus wurde schließlich nahezu unverändert übernommen, nämlich die zweigeteilte Exekutive und das Kollegialprinzip innerhalb der Regierung. Mit ihrer Hilfe sollte die Macht des Premierministers institutionell begrenzt werden.

Die Tel Aviver Professoren knüpften an das "präsidentiell-parlamentarische« System hohe Erwartungen. Bernard Susser, einer der Mitautoren des Entwurfs, sah in ihm ein Modell, das nicht nur für Israel Gültigkeit hatte, sondern in der Bedeutung darüber hinaus reichte. Seine Hoffnung war, dass die neue Regierungsform die Stabilitätseigenschaften des Präsidentialismus mit der größeren Anpassungsfähigkeit eines parlamentarischen Systems verbinden könnte. Die Vetomacht der kleinen 
Parteien sollte eingeschränkt, ihre legitime Rolle im politischen Prozess aber nicht in Frage gestellt werden. Die Stärkung des Premierministers würde dafür sorgen, dass die Exekutive auch dann noch handlungsfähig war, wenn sie im Parlament über keine feste Mehrheit verfügte. Gleichzeitig enthielt das System genügend Sicherungen, um einem möglichen Machtmissbrauch des Regierungschefs vorzubeugen. Susser verhehlte nicht, dass die Kombination von direkter Volkswahl und parlamentarischer Abberufbarkeit des Premierministers aus logischer Sicht schwer vermittelbar war, ja eine Zumutung darstellte. Unter den besonderen Umständen der israelischen Politik schien sie ihm jedoch der am besten geeignete Weg, die Kriterien der Effektivität (Regierbarkeit) und Demokratie in eine vernünftige Balance zu bringen. ${ }^{46}$

\section{d) Das Reformgesetz von 1992}

Eine modifizierte Form des Reichman-Entwurfes wurde im September 1990 von Amnon Rubinstein und drei weiteren Abgeordneten als Gesetzesvorlage eingebracht und anschließend im Rechts- und Verfassungsausschuss der Knesset beraten. Die Änderungen gegenüber dem ursprünglichen Konzept betrafen u.a. den Zeitpunkt der Wahl des Premierministers (diese sollte nun schon im ersten Wahlgang zusammen mit den Parlamentswahlen erfolgen) und das Inkompatibilitätsgebot (das komplett gestrichen wurde). Des Weiteren sah der Entwurf - in einer Anleihe aus dem amerikanischen System - die Einrichtung des Amtes eines stellvertretenden Premierministers vor. Dieser sollte den Regierungschef nach dessen Tod, Rücktritt oder Amtsenthebung für den Rest der Legislaturperiode automatisch beerben. Eine Amtsenthebung konnte von der Knesset mit einfacher Mehrheit beschlossen werden, wenn der Premierminister (aus gesundheitlichen Gründen) handlungsunfähig war oder sich eines moralischen Fehlverhaltens schuldig gemacht hatte.

Während die oppositionelle Arbeitspartei den Reformplan geschlossen unterstützte, hegte der Likud-Block unter Premierminister Schamir grundsätzliche Vorbehalte gegen das Direktwahlkonzept. Der Entwurf musste daher vor der entscheidenden Abstimmung zur erneuten Beratung in den Rechts- und Verfassungsausschuss überwiesen werden, nachdem er schon in der zweiten Lesung um ein Haar durchgefallen wäre. Während der Ausschusssitzungen gelang es den $L i-$ $k u d$-Vertretern im Verein mit der Nationalreligiösen Partei, weitere Änderungen durchzusetzen, die dem Direktwahlkonzept die »Giftzähne« ziehen sollten. Kritiker bezeichneten dieses Vorgehen später als "zynischen Akt des Protestes «, dessen einziger Zweck es gewesen sei, das Reformwerk zu entwerten. ${ }^{47}$ Dennoch waren die Befürworter am Ende bereit, der veränderten Vorlage zuzustimmen, um ein Inkrafttreten des Gesetzes rechtzeitig zur nächsten Legislaturperiode zu ermöglichen. ${ }^{48}$

46 Ebd.

47 Diskin / Diskin, aaO. (FN 34), S. 42.

48 Vgl. Keller, aaO. (FN 36), S. 612ff.

ZfP 53.Jg. 3/2006 
Das von der Knesset am 18. März 1992 beschlossene »Grundgesetz: Die Regierung « unterschied sich von dem Entwurf des Reichman-Komitees in folgenden Punkten:

1) Die Wahl des Premierministers setzte in beiden Wahlgängen eine absolute Mehrheit voraus, wobei der erste Wahlgang zeitgleich mit den Parlamentswahlen stattfinden sollte. (Diese Änderung war bereits in der 1990 eingebrachten Gesetzesvorlage enthalten.)

2) Die vom Premierminister gebildete Regierung musste innerhalb von 45 Tagen durch die Knesset mit absoluter Mehrheit (50 Prozent +1 ) bestätigt werden. Bei Nicht-Zustandekommen dieser Mehrheit waren gleichzeitige Neuwahlen für Parlament und Premierminister vorgesehen. Dasselbe galt für den Fall eines regulären Misstrauensvotums, das ebenfalls bereits mit absoluter Mehrheit beschlossen werden konnte. Eine Auflösung des Parlaments durch den Premierminister war weiterhin möglich, erforderte jedoch die Zustimmung des Staatspräsidenten. Auch danach würde es zu gleichzeitigen Neuwahlen des Parlaments und Premierministers kommen. Eine Neuwahl (special election) nur des Premierministers hatte stattzufinden, falls dieser während der drei ersten Jahre der Legislaturperiode starb, freiwillig zurücktrat, amtsunfähig war oder seines Postens von der Knesset enthoben wurde, wobei letzteres die Zustimmung von mindestens 80 Abgeordneten erforderte. Trat eines der genannten Ereignisse im letzten Jahr der Wahlperiode ein, waren gleichzeitige Neuwahlen für Parlament und Premier vorgesehen.

3) Für den Bereich der Regierung war vorgeschrieben, dass mindestens die Hälfte der (maximal 18) Minister zugleich Abgeordnete der Knesset sein mussten. Das Inkompatibilitätsgebot des Reichman-Entwurfes wurde damit praktisch in sein Gegenteil verkehrt, was bei den Urhebern des ursprünglichen Reformvorschlags Befremden hervorrief. Auf einhellige Zustimmung stieß hingegen eine dem Gesetz neu hinzugefügte Regelung, die es Partei- und Fraktionswechslern untersagte, während der laufenden Legislaturperiode ein Ministeramt zu übernehmen. Den Abgeordneten sollte auf diese Weise der Anreiz genommen werden, ihr Mandat um des eigenen Vorteils willen $\mathrm{zu} »$ verkaufen«.

Mit dem Sieg der Arbeitspartei bei den - noch unter dem alten Recht durchgeführten - Parlamentswahlen endete 1992 die 15-jährige Regierungszeit des Likud. Die von Jitzchak Rabin angeführte neue Regierung unternahm in der Folge mehrere Anläufe, das Direktwahlgesetz im Sinne des ursprünglichen Entwurfs zu korrigieren, die aber alle keinen Erfolg brachten. Die Urheber des »präsidentiell-parlamentarischen « Systems stießen sich insbesondere an drei Punkten: Erstens monierten sie, dass die Hürde für ein erfolgreiches Misstrauensvotum in dem beschlossenen Gesetz von 73 auf 61 Stimmen gesenkt worden war, was den Handlungsspielraum des Premierministers verkleinerte und eine Fortschreibung der bereits bestehenden Regelung bedeutete. Zweitens wandten sie sich aus demselben Grund dagegen, dass die Regierung vor ihrem Amtsantritt der Bestätigung durch die Knesset bedurfte. In seiner 1989 veröffentlichten theoretischen Abhandlung hatte Bernard Susser eine solche Konstruktion als widersinnig ausgeschlossen: »[I]f a popularly elected Chief Executive could be removed from office by a hostile legislature the morning after 
his / her victory, it would mean nothing less than the destruction of the political system. It is not surprising ... that such a system has nowhere been adopted « ${ }^{49}$. Drittens schließlich wurde der Verzicht auf die in der Vorlage der vier Knessetabgeordneten enthaltene Stellvertreterregelung kritisiert. Dies führte dazu, dass in der neuen Fassung zahlreiche Vorkehrungen für Neuwahlen (des Parlaments und Premiers bzw. nur des Premiers) getroffen werden mussten, die geeignet waren, das Konfliktpotenzial zwischen Exekutive und Legislative zu erhöhen..$^{50}$ Zusammengenommen liefen die Änderungen auf eine Verstärkung bzw. Wiederherstellung der parlamentarischen Abhängigkeit des Regierungschefs hinaus, die ja durch die Einführung der Direktwahl gerade reduziert werden sollte. Die Reformer waren daher skeptisch, ob das Gesetz in der beschlossenen Form imstande sein würde, die Durchsetzungsmacht des Premierministers gegenüber den religiösen Parteien tatsächlich zu steigern. ${ }^{1}$

\section{Die kontraproduktiven Auswirkungen der Direktwabl}

Die Erfahrungen mit dem neuen System sollten die schlimmsten Befürchtungen der Kritiker noch übertreffen. Sie führten dazu, dass der präsidentielle Parlamentarismus in Israel nur sieben Jahre Bestand hatte. Schon 2001 beschloss die Knesset, ab der nächsten Wahl zu einer modifizierten Form der rein parlamentarischen Regierungsweise zurückzukehren, was dann - nach erneut vorgezogenen Neuwahlen im Jahre 2003 auch geschah. Die Wiedereinsetzung des alten Systems wurde ohne langwierige Beratungen in einem parlamentarischen Schnellverfahren gleichsam durchgepeitscht, so als ob die Abgeordneten das Scheitern der Verfassungsreform möglichst rasch vergessen machen wollten. Die unintendierten Konsequenzen der Direktwahl, die den Vorstellungen der Reformer diametral entgegenliefen, lassen sich dabei auch am Abstimmungsverhalten ablesen: Wurde die Einführung des präsidentiell-parlamentarischen Systems 1992 von Arbeitspartei und Likud gegen den Widerstand der kleineren Parteien durchgesetzt, so waren es jetzt die kleinen Parteien (die religiösen wie die säkularen), die der Rückkehr zum reinen Parlamentarismus widersprachen. ${ }^{52}$

Unter der Gültigkeit der 1992 beschlossenen Reform wurde der israelische Premierminister dreimal (1996, 1999 und 2001) direkt vom Volk bestellt. Alle drei Wahlen endeten mit einer Niederlage des jeweiligen Amtsinhabers. Fiel die Niederlage für Schimon Peres, den parlamentarisch bestellten Nachfolger Jitzchak Rabins, 1996 noch äußerst knapp aus, so wurden die beiden volksgewählten Premiers, Benjamin Netanjahu und Ehud Barak, von den Wählern 1999 und 2001 regelrecht davongejagt. Das Sanktionswahlverhalten ging dabei soweit, dass letzterer 2001 nahezu die

49 Susser, aaO. (FN 43), S. 112.

50 Keller, aaO. (FN 36), S. 617.

51 Diskin / Diskin, aaO. (FN 34), S. 42f.

52 Emanuele Ottolenghi, »Why Direct Election Failed in Israel» in: Journal of Democracy 12 (2001) H.4, S. 121. 
Hälfte (!) seiner vormaligen Wähler verlor. Baraks Nachfolger Ariel Scharon gelang demgegenüber unter dem wiederhergestellten rein parlamentarischen System 2003 ein eindrucksvoller Wahlsieg, als seine Likud-Partei mit großem Abstand stärkste Kraft wurde und die Zahl der Mandate in der Knesset im Vergleich zur Parlamentswahl 1999 verdoppeln konnte..$^{53}$

Die starken Wählerbewegungen sind keine unmittelbare Folge des Direktwahlsystems, sondern müssen vor dem Hintergrund der dramatischen Ereignisse gesehen werden, die Israels Politik und Gesellschaft seit dem Jahre 2000 überschatteten. War Baraks Triumph über Netanjahu 1999 noch Ausdruck der Hoffnung gewesen, den mit dem Oslo-Abkommen eingeleiteten Friedensprozess beschleunigen und zu einem Ausgleich mit den Palästinensern kommen zu können, so markierte der Sieg des »Falken« Ariel Scharon das vorläufige Ende dieser Bemühungen. Das Scheitern des Dreier-Gipfels in Camp David, wo eine Einigung zwischen Barak und Arafat in greifbare Nähe gerückt schien, und die kurz darauf ausgebrochene »Al Aksa-Intifada« in den Palästinensergebieten führten dazu, dass der Premierminister 2001 in sämtlichen Regionen des Landes und Wählergruppen gewaltig an Zustimmung einbüßte. Am größten waren seine Verluste unter den arabischen Israelis, die Barak 1999 noch zu 95 Prozent unterstützt hatten, nun aber die Wahl praktisch boykottierten (ihre Wahlbeteiligung sank von 75 auf 18 Prozent), während auf der anderen Seite ein großer Teil der jüdischen Wähler Baraks direkt zu Scharon überliefen. ${ }^{54}$

Die Einschätzung der (unmittelbaren) Auswirkungen der Direktwahl wird auch durch die längerfristige Entwicklung des Wählerverhaltens erschwert, die in Israel von zwei scheinbar gegenläufigen Tendenzen charakterisiert ist. Einerseits kommt es zu einer Abschwächung überkommener Parteibindungen, die sich in zunehmender Wahlabstinenz und Wechselbereitschaft niederschlägt - eine Tendenz, die uns aus den meisten westlichen Demokratien wohlvertraut ist. Auf der anderen Seite hat die dadurch beförderte Fragmentierung nicht zu einer Entideologisierung geführt; die Polarisierung der israelischen Politik und Parteienlandschaft wurde im Gegenteil in den neunziger Jahren weiter verstärkt. Auch hier lassen sich vergleichbare Entwicklungen eines Bedeutungsgewinns kultureller oder wertebezogener Konflikte in anderen Ländern ausmachen, die dort allerdings nicht primär unter religiösen Vorzeichen ablaufen (mit der charakteristischen Ausnahme der USA). In Israel ist die Konfliktlinie Religion / Staat für das Parteiensystem hingegen immer prägender geworden. Bis 1992 nur von untergeordneter Bedeutung, bestimmt sie das Wahlverhalten seit 1996 stärker als die sozial-ökonomischen Fragen. ${ }^{55}$ Symptomatisch dafür ist der Aufstieg der Partei Shinui (»Wandel«), die der linksliberalen Meretz als Sachwalterin des säkularen Israel und Gegengewicht zu den religiösen Parteien erfolgreich den Rang ablaufen konnte und inzwischen zur drittstärksten politischen Kraft des Landes avanciert ist.

53 Vgl. Cameron S. Brown, »Israel's 2003 Elections. A Victory for the Moderate Right and Secular Center« in: Middle East Review of International Affairs 7 (2003) H.1, S. 77-94.

54 Vgl. Abraham Diskin / Reuven Y. Hazan, "The 2001 Prime Ministerial Election in Israel in: Electoral Studies 21 (2002) H.4, S. 659-664.

55 Vgl. Asher Arian / Michael Shamir, »Candidates, Parties and Blocs. Israel in the 1990s « in: Party Politics 7 (2001) H.6, S. 689-710. 
Die Hoffnung der Direktwahlbefürworter bestand darin, dieser Tendenz einer weiteren Fragmentierung und Polarisierung des Parteiensystems entgegenwirken zu können. Der präsidentielle Parlamentarismus sollte das System einerseits demokratischer machen, indem er das Zustandebringen einer neuen Mehrheit nicht mehr einseitig den Politikern überließ, sondern an ein vorgängiges Votum des Volkes band, und andererseits seine Regierungsfähigkeit erhöhen. Von beiden Zielen konnte nur das erste erreicht werden. Die demokratisierende Wirkung lässt sich daran festmachen, dass die Wahlen, die nach Einführung des neuen Systems stattfanden, allesamt vorgezogen werden mussten, was von 1948 bis 1996 insgesamt nur dreimal der Fall gewesen war. $2001 \mathrm{kam}$ es dabei zu einer sogenannten »special election«, während 1999 und 2003 sowohl der Premierminister als auch das Parlament neu bestimmt wurden. Die verkürzten Wahlzyklen sind kein Ausdruck einer größeren Instabilität der israelischen Politik, da es Regierungswechsel und -umbildungen auch zu früheren Zeiten häufig gegeben hatte - nur wurden diese in der Regel eben ohne Beteiligung des Wählers herbeigeführt. ${ }^{56}$

Das Mehr an Demokratie auf der Input-Seite fällt jedoch kaum ins Gewicht, da die Reform das andere große Ziel verfehlte. Hier erreichte man mit der Direktwahl genau das Gegenteil des Erhofften: Statt die Regierungsfähigkeit des Premierministers gegenüber dem Parlament zu erhöhen, führte das System dazu, dass es für den Regierungschef schwieriger wurde, eine einigermaßen stabile Mehrheitskoalition in der Knesset zu bilden und zusammenzuhalten. Nachdem die Direktwahl 1996 zum ersten Mal durchgeführt wurde, konnten die Befürworter deren kontraproduktive Wirkungen noch auf das strategische Unvermögen der politischen Akteure zurückführen, sich der Funktionslogik des präsidentiell-parlamentarischen Systems anzupassen. ${ }^{57}$ Als 1999 und 2001 immer noch keine Besserung eintrat und die unintendierten Konsequenzen des Direktwahlsystems offen zu Tage traten, mussten sie das Scheitern der Reform eingestehen.

Der Hauptirrtum der Reformer lag in der Erwartung, dass es durch die Gleichzeitigkeit von Premier- und Parlamentswahl zu einer engen politischen und elektoralen Koppelung der Urnengänge kommen würde. Dies wurde in doppelter Hinsicht widerlegt.

Erstens konzentrierte sich die Aufmerksamkeit der Wählerschaft und beiden groBen Parteien ganz auf die Direktwahl des Premiers, sodass man den Eindruck gewinnen konnte, die Parlamentswahlen spielten nur eine untergeordnete Rolle oder würden gar nicht stattfinden. Die »Präsidentialisierung«, die in Israel schon vorher zu einer kumulativen Aufwertung der Funktion des Regierungschefs geführt hatte, tat hier ihre Wirkung. ${ }^{58}$ Sie schlug sich in einem betont überparteilichen Wahlkampf

56 Vgl. Emanuele Ottolenghi, »Immobility, Stability and Ineffectiveness. Assessing the Impact of Direct Election of the Prime Minister in: Journal of Legislative Studies 5 (1999) H.1, S. 44f.

57 Vgl. Bernard Susser, »The Direct Election of the Prime Minister. A Balance Sheet « in: Israel Affairs 4 (1997) H.1, S. 248.

58 Vgl. Reuven Y. Hazan, "The Failure of Presidential Parliamentarism. Constitutional versus Structural Presidentialization in Israel's Parliamentary Democracy « in: Thomas Poguntke / Paul Webb (Hg.), The Presidentialization of Politics, Oxford/New York 2005, S. 289-312. 
der Kandidaten für das Amt des Premierministers nieder, die - wenn sie erfolgreich sein wollten - um die Unterstützung der Wähler aus der politischen Mitte buhlen mussten und dafür auf deren »Parteistimme bei den Knessetwahlen stillschweigend oder ausdrücklich verzichteten (indem den kleineren Parteien durch Wahlabsprachen Listenplätze zugesichert wurden). Dem Zwang, populäre und mehrheitsfähige Kandidaten zu nominieren, trugen dabei auch die von der Arbeitspartei 1992 erstmals eingesetzten primaries Rechnung, von denen sich die Befürworter der Reform zugleich eine Lockerung der Fraktionsdisziplin im Parlament versprachen..$^{59}$

Zweitens sorgten die unterschiedlichen Verfahren bei der Premier- und Parlamentswahl dafür, dass die ersteren von den beiden großen Parteien vollständig monopolisiert wurden. Die Kandidaten von Likud und Arbeitspartei konnten so bei allen drei Direktwahlen das Rennen unter sich ausmachen. Vertreter kleinerer Parteien traten entweder erst gar nicht an oder zogen ihre Kandidatur (wie 1999) vor der Wahl zurück, sodass es - anders als von den Reformern vermutet - in keinem der Fälle zu einer Stichwahl kam. Diese hätte dann im Lichte des bereits vorliegenden Ergebnisses der Parlamentswahlen stattfinden müssen und damit dem Kandidaten der siegreichen Partei womöglich einen Vorteil verschafft. So aber mussten sich die Vertreter der kleineren Parteien vor einer klaren Festlegung auf einen der Kandidaten im Vorfeld der Wahl hüten, um nicht Gefahr zu laufen, auf das falsche Pferd zu setzen. ${ }^{60}$ Die dadurch ermöglichte Flexibilität erhöhte zwar die Chancen des gewählten Premiers, eine Mehrheit in der Knesset hinter sich zu bringen; sie bot aber keine Gewähr für eine dauerhafte parlamentarische Unterstützung.

Hatten sich die Reformer von der Gleichzeitigkeit der Premier- und Parlamentswahlen einen »coattail«-Effekt (nach US-amerikanischen Vorbild) erwartet, bei dem der siegreiche Direktwahlkandidat die Kandidaten seiner Parlamentspartei mit sich ziehen würde, so trat in der Praxis genau das Gegenteil ein. Die Wähler nutzten das neue System, um ihre Stimme zu »splitten «. Weil sie sich bei der Wahl des Premiers auf einen Kandidaten der beiden großen Parteien festlegen mussten, konnten sie bei der als minder wichtig betrachteten Parlamentswahl um so ungenierter für die kleineren Vertreter votieren. Schon bei der ersten Doppelwahl 1996 machten von dieser Möglichkeit nicht weniger als 45 Prozent der Wähler Gebrauch. ${ }^{61}$ Die gleichzeitige Wahl von Regierungschef und Parlament bot ihnen einen Anreiz, ihre Stimme nach unterschiedlichen Gesichtspunkten zu vergeben. »Während bei der Wahl des Premiers die weltanschauliche Grundorientierung und natürlich die Bewertung der konkreten Kandidaten den Ausschlag gaben, lud die beibehaltene Listenwahl zur Stimmabgabe für diejenige Partei ein, die die wirksamste Vertretung der eigenen Partikular- und Gruppeninteressen versprach «62 . Auch hier hätte es sich für die Reformer lohnen können, die US-amerikanischen Erfahrungen etwas gründlicher zu

59 Vgl. Keller, aaO. (FN 36), S. 623.

60 Vgl. Susser, aaO. (FN 57), S. 246.

61 Vgl. Ottolenghi, aaO. (FN 56), S. 46.

62 Michael Edinger, »Nachwehen des premier-parlamentarischen Intermezzos. Die Knessetwahl 2003 vor dem Hintergrund einer gescheiterten Verfassungsreform « in: Zeitschrift für Parlamentsfragen 34 (2003) H.2, S. 339. 
studieren, wo das policy-bezogene »ballot splitting « als eine der Hauptursachen von »divided government « betrachtet wird.

Konsequenz des Stimmensplittings war, dass die Fragmentierung des Parteiensystems zunahm und die kleinen Parteien, deren Einfluss durch die Reform gerade beschnitten werden sollte, bei den Parlamentswahlen immer stärkeren Zulauf erfuhren. Bestand die Knesset 1992 aus 10 Fraktionen, so waren es 199611 und 1999 bereits 15. Der Stimmenanteil der religiösen Parteien wuchs unterdessen um das Doppelte (von 14 auf 23 bzw. 27 Sitze), während die von den beiden größten Parteien gehaltenen Mandate von 76 (1992) auf 66 (1996) bzw. 45 (1999) zurückgingen. Betrachtet man die Gesamtstärke der sogenannten »Sektorparteien $\ll^{63}$, so lag diese 1999 mit 50 Mandaten höher als der zusammengenommene Anteil von Likud und Arbeitspartei. Auch eine Große Koalition verfügte zu diesem Zeitpunkt also über keine eigene Mehrheit mehr und bedurfte, als sie 2001 geschlossen wurde, der Unterstützung durch gleich mehrere Koalitionspartner.

Abb.: Knessetwablen 1973 bis 2003. Quelle: Edinger, aaO. (FN 62), S. 342.

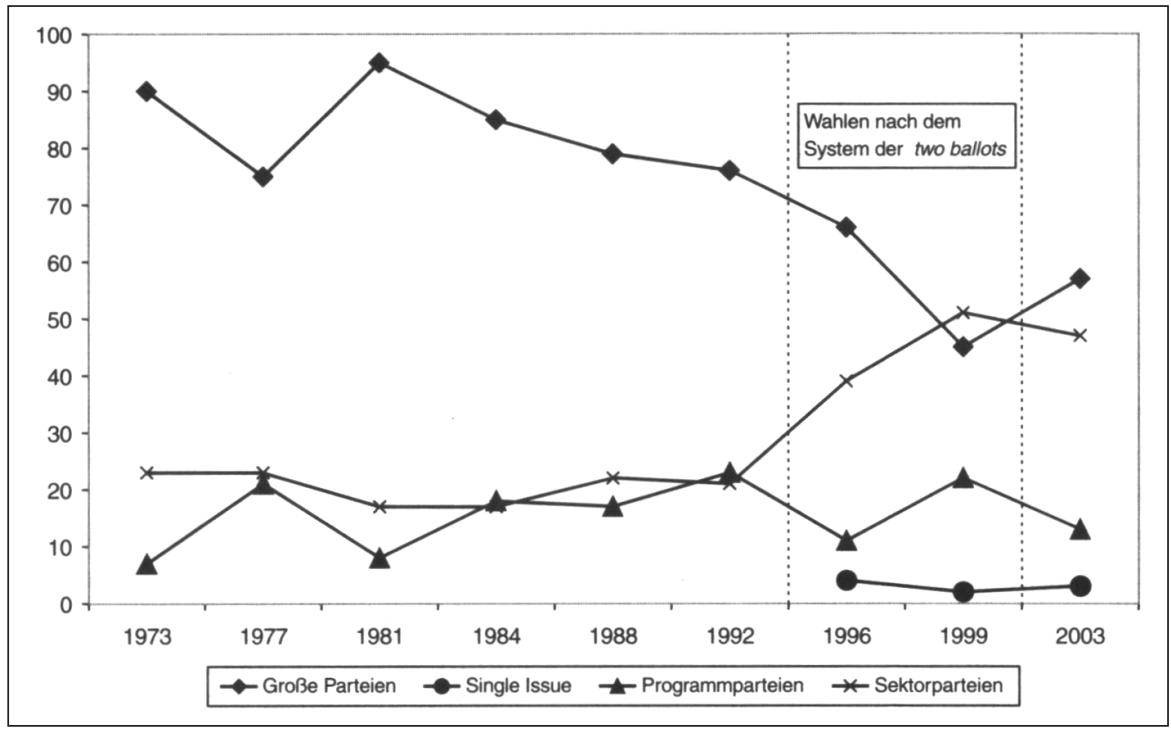

Nachdem die Fragmentierung und Polarisierung des Parteiensystems durch die Direktwahl zusätzlich befördert wurde, waren die nach 1996 gebildeten Koalitionsregierungen wesentlich disparater und fragiler als ihre Vorgängerinnen. So musste

63 Hierunter werden Parteien verstanden, die ihre Unterstützung aus einem spezifischen, sozialstrukturell abgrenzbaren Bevölkerungsklientel beziehen. In Israel gehören dazu zum einen die religiösen Parteien und ihre laizistischen Gegenspieler, zum anderen die ethnischen bzw. Einwandererparteien. Von diesen zu unterscheiden sind die nach ideologischen Kriterien definierten »Programmparteien«. Ebd., S. $339 \mathrm{f}$. 
der mit hauchdünnem Vorsprung zum Premier gewählte Benjamin Netanjahu in seine Koalition auch Vertreter der zum linken Block gehörenden Gescher-Partei mit einbeziehen, die ihm Anfang 1998 nach einem Haushaltsstreit prompt den Rücken kehrten und die Regierungsmehrheit auf 62 von 120 Mandaten zusammenschmelzen ließen. Als es dann auch noch in seiner eigenen Likud-Partei zur offenen Auseinandersetzung über das mit Palästinenserchef Arafat ausgehandelte Wye-Abkommen kam, war das Schicksal des Premiers besiegelt. ${ }^{64}$ Aus den vorgezogenen Neuwahlen ging der Kandidat der Arbeitspartei, Ehud Barak, als klarer Gewinner hervor. Gemessen an dem Ergebnis der Parlamentswahlen handelte es sich aber auch hier um einen Pyrrhussieg. Obwohl das unter dem Namen "Jisrael Achat « (Ein Israel) angetretene Bündnis von Arbeitspartei, Gescher und Meimad gegenüber dem Likud die Oberhand behalten hatte, konnte es nur noch gut ein Viertel der Parlamentarier hinter sich versammeln - nie zuvor war eine Regierungsfraktion in der israelischen Politik zahlenmäßig schwächer gewesen. Eine durch Grundgesetzänderung beschlossene Aufstockung der Kabinettsposten half Barak, eine Koalition aus insgesamt sieben Parteien zu bilden, die über 75 Knessetsitze verfügte. ${ }^{65}$ Die gleichzeitige Einbeziehung der Exponenten des säkularen und ultrareligiösen Lagers ( $M e-$ retz und Schas-Partei) machte diese Koalition jedoch noch heterogener und brüchiger als ihre Vorgängerin. Der Erosionsprozess begann bereits einen Tag nach der Vereidigung, als zwei Abgeordnete der russischen Einwandererpartei Jisrael Ba-Ali$j a h$ das Regierungsbündnis verließen, und setzte sich bis zum Sommer 2000 fort kurz bevor Barak in die USA aufbrach, um mit Arafat über die Gründung eines Palästinenserstaates zu verhandeln. Ungeschicklichkeiten im Umgang mit den Koalitionspartnern und das Scheitern des Gipfels führten dazu, dass nach etwas mehr als einem Jahr im Amt nur noch zwei Fraktionen mit 30 Abgeordneten hinter dem Regierungschef standen. Obwohl in dieser Situation eigentlich eine komplette Neuwahl angezeigt gewesen wäre, entschied sich Barak, eine solche lediglich für das Amt des Premierministers anzustreben. Das Direktwahlgesetz eröffnete ihm diese Möglichkeit durch eigenen Rücktritt. Auf diese Weise hoffte er nicht nur die voraussehbare Niederlage bei einer möglichen Parlamentswahl zu umgehen, sondern auch eine erneute Kandidatur seines 1999 unterlegenen Rivalen Netanjahu, der als Nicht-Mitglied der Knesset nur bei einer Doppelwahl hätte antreten dürfen.6 ${ }^{66}$ Das Kalkül nützte dem Premier allerdings nicht viel. Nach mehr als einem halben Jahr lähmenden politischen Stillstands hatte sein Ansehen in der israelischen Öffentlichkeit so gelitten, dass auch Ariel Scharon, dem im Vergleich zu Netanjahu die gerin-

64 Vgl. Reuven Y. Hazan / Abraham Diskin, »The 1999 Knesset and Prime Ministerial Elections « in: Electoral Studies 19 (2000) H.4, S. 628-646.

65 Angelika Timm, Israel. Gesellschaft im Wandel, Opladen 2003, S. 128.

66 Barak hatte zwar seine Unterstützung für eine Gesetzesänderung signalisiert, die Netanjahu die Kandidatur bei einer "special election « ermöglichen würde. Er verweigerte sich jedoch dessen Forderung nach gleichzeitigen Parlamentswahlen. Mit Blick auf die Mehrheitsverhältnisse in der weiter bestehenden Knesset zog es Netanjahu darauf hin vor, auf seine Kandidatur zu verzichten. Diskin / Hazan, aaO. (FN 54), S. 661f. 
geren Chancen eingeräumt worden waren, leicht über ihn triumphieren und im Februar 2001 einen haushohen Wahlsieg einfahren konnte.

Da die Likud-Partei des neu gewählten Premiers in der bestehenden Knesset noch nicht einmal ein Sechstel der Sitze hielt, entschied Scharon, eine »große « Koalition mit der Arbeitspartei einzugehen, in die weitere fünf Parteien, vorzugsweise aus dem religiösen Lager, aufgenommen wurden. Eine der ersten Amtshandlungen der neuen Regierung bestand darin, das Direktwahlgesetz aufzuheben und zur alten parlamentarischen Regierungsform zurückzukehren (wenn auch mit einigen Veränderungen). ${ }^{67}$ Wussten sich Arbeitspartei und Likud in dieser Frage vollkommen einig, so gestaltete sich ihre Zusammenarbeit ansonsten eher schwierig. Von daher kam es nicht überraschend, dass auch die Große Koalition nach rund eineinhalb Jahren platzte. Auseinandersetzungen um die Siedlungspolitik und der Führungsstreit in der Arbeitspartei sorgten dafür, dass die Israelis im Januar 2003 erneut vorzeitig zu den Urnen gerufen werden mussten. Die unter dem wiederhergestellten alten System mit nur einer Listenstimme durchgeführten Wahlen bescherten Scharons Likud einen unerwartet deutlichen Wahlsieg. Der Premier konnte dabei mit den 38 Abgeordneten seiner Partei mehr Parlamentarier hinter sich versammeln als die drei durch Direktwahl ins Amt gelangten Regierungschefs vor ihm. Weil sich die Arbeitspartei der von Scharon gewünschten Neuauflage einer Regierung der nationalen Einheit verweigerte, bildete der Likud zusammen mit Shinui, der Nationalreligiösen Partei und der Nationalistischen Rechten (Ichud Le'umi) daraufhin eine Viererkoalition, an der zum ersten Mal seit langer Zeit keine orthodoxen Vertreter beteiligt waren. Die Wiedereinsetzung des rein parlamentarischen Systems bewirkte einen Rückgang der Fragmentierung in der Knesset, doch lag die Zahl der Fraktionen mit 12 (statt 15) immer noch höher als vor Einführung der Direktwahl. Wichtiger war, dass die größte Fraktion jetzt auch innerhalb der Regierung wieder die Oberhand hatte und mehr als die Hälfte der Minister stellen konnte. Der Premierminister verfügte dadurch über eine stärkere Position als seine direkt gewählten Vorgänger. ${ }^{68}$ Dies verhinderte zwar nicht, dass die Regierung nach zwei Jahren im Amt ebenfalls in heftige Turbulenzen geriet (an denen sie schließlich zerbrechen sollte), doch lag dies vor allem an der Tragweite der von Scharon betriebenen Politik, dessen Entscheidung, die israelischen Siedlungen im Gaza-Streifen aufzulösen, erheblichen Widerstand in den eigenen Reihen hervorrief und nur mit Unterstützung der Arbeitspartei im Parlament durchgesetzt werden konnte. Unter dem Strich besteht kein Zweifel, dass sich die Rückkehr zum reinen Parlamentarismus ausgezahlt und zu einer verbesserten Regierungsfähigkeit des Systems beigetragen hat.

67 Die Änderungen betrafen zum einen das Recht der Parlamentsauflösung durch den Premierminister, das aus dem präsidentiell-parlamentarischen System übernommen wurde; zum anderen entschloss man sich zur Einführung eines konstruktiven Misstrauensvotums nach deutschem Vorbild.

68 Edinger, aaO. (FN 62), S. 344f. 


\section{Schlussbemerkung}

Der präsidentielle Parlamentarismus in Israel ist ein spektakuläres Beispiel für die unintendierten Konsequenzen institutioneller Reformen. Die konstitutionelle Aufwertung des Regierungschefs, die das Direktwahlsystem herbeiführen wollte, bewirkte in der Praxis seine faktische Schwächung, also das glatte Gegenteil. Auch die Befürworter der Reform mussten deshalb zugeben, dass der Versuch einer Synthese der parlamentarischen und präsidentiellen Regierungsform grandios gescheitert war.

Bleibt die Frage, ob dieses Scheitern den spezifischen Konstruktionsmängeln des von den Vorstellungen der Reformer in wesentlichen Punkten abweichenden Direktwahlgesetzes angelastet werden muss, oder ob es nicht vielmehr bereits in der Grundkonzeption des präsidentiellen Parlamentarismus angelegt war, der den Versuch darstellte, zwei miteinander unvereinbare Konstruktionsprinzipien politischer Systeme zu verbinden? Im Kontext der israelischen Politik deutet die Antwort eher in die zuletzt genannte Richtung. Das Experiment wäre also vermutlich ähnlich ausgegangen, wenn man dem ursprünglichen Entwurf des Reichman-Komitees gefolgt wäre, der im Vergleich zu dem später verabschiedeten Gesetz eine stärkere Betonung der präsidentiellen Komponente vorgesehen hatte.

Betrachten wir dazu zunächst das Problem der zeitlichen Koppelung von Premier- und Parlamentswahlen. Die Reformer waren sich der Tatsache bewusst, dass die Regierungsfähigkeit des Premiers entscheidend davon abhängen würde, ob er im Parlament gleichgerichtete Mehrheitsverhältnisse vorfindet. Diesen Effekt wollten sie durch die gleichzeitige Ansetzung der Parlamentswahl mit der (vermutlich wahlentscheidenden) zweiten Runde der Premierwahlen befördern. Die von ihnen nicht vorausgeahnte deutliche Höherbewertung der Premierwahl bewirkte jedoch das Gegenteil. Sie erzwang eine Entscheidung bereits im ersten Wahlgang und gab den Wählern einen Anreiz, ihre Stimmen aufzuteilen. Dies ist nicht ohne Ironie, wenn man den ursprünglichen Entwurf des Reichman-Komitees betrachtet. Wäre die Entscheidung über den Premier bereits im ersten Wahlgang gefallen, hätten die Parlamentswahlen nach diesem Entwurf ja zeitlich versetzt stattgefunden. Gerade das hätte dem gewählten Premier aber die Möglichkeit gegeben, die notwendige Koalitionsbildung zu betreiben und die Wähler bei der Parlamentswahl in seine Richtung mitzuziehen. Der Effekt wäre derselbe gewesen wie im semi-präsidentiellen Frankreich, wo die Präsidentschaftswahlen den Wahlen zur Nationalversammlung stets vorausgehen und ein gewählter Präsident durch seine Auflösungsbefugnis eine ihm gewogene parlamentarische Mehrheit »erzeugen« kann. Eine Umsetzung des Reichman-Entwurfs hätte den gewünschten »Gleichschaltungseffekt» also tatsächlich herbeiführen und damit zum besseren Funktionieren des Systems beitragen können - wenn auch auf ganz andere Weise, als von den Reformern geplant.

Die Bedeutung der übrigen Modifikationen muss demgegenüber geringer veranschlagt werden. Zwar hatten die Befürworter des Reichman-Entwurfs nicht unrecht, die nachträglich eingeführte Investiturabstimmung, die Absenkung des Quorums für das Misstrauensvotum und die diversen Vorkehrungen für »special 
elections « als Schwächung der Position des Premierministers zu beklagen. Fraglich ist aber, ob die Übernahme dieser Elemente, mit denen die Balance des Systems etwas mehr in Richtung Präsidentialismus verschoben worden wäre, dessen Funktionsfähigkeit wirklich substanziell verbessert hätte. Das Kardinalproblem des präsidentiell-parlamentarischen Systems lag von Beginn an in der bleibenden Abhängigkeit des Premiers von einer ihn unterstützenden parlamentarischen Mehrheit, die durch die nachträglichen Korrekturen allenfalls bekräftigt wurde. Einige Autoren haben deshalb die Charakterisierung des 1996 eingeführten Systems als Mischtyp überhaupt in Zweifel gezogen und die Direktwahl als lediglich supplementäres Element der parlamentarischen Regierungsform bezeichnet, die in Israel weiterhin fortbestanden habe. ${ }^{69}$ Selbst wenn man diese Interpretation für das ursprüngliche Modell nicht teilt, wäre das Scheitern des präsidentiellen Parlamentarismus in Israel so oder so vorprogrammiert gewesen. Eine noch größere Unantastbarkeit des Premierministers, wie sie der Reichman-Entwurf angestrebt hatte, hätte diesen ja nicht von der Notwendigkeit befreit, eine parlamentarische Mehrheit für seine Politik zu organisieren. Unter den in Israel obwaltenden Bedingungen wäre das weitergehende Modell sogar nachteiliger gewesen als das tatsächlich verabschiedete Gesetz, weil es die Gefahr einer dauerhaften Blockade zwischen Parlament und Regierung erhöht hätte.

Parlamentarisch-präsidentielle Mischsysteme müssen entweder einen annähernden politischen Gleichklang zwischen beiden Organen sicherstellen oder dafür Sorge tragen, dass die Regierungsfähigkeit auch bei abweichenden Mehrheitsverhältnissen erhalten bleibt. Beide Anforderungen konnten in Israel nicht erfüllt werden. Scheiterte die Erzeugung synchroner Mehrheiten an den institutionellen Konstruktionsmängeln des Systems, so fehlte es für ein funktionierendes »divided govern$m e n t$ « bereits an den prinzipiellen Voraussetzungen. Diese liegen, wie das US-amerikanische Beispiel zeigt, in einer weitgehenden Zurückdrängung der "partisan politics «, organisatorisch und ideologisch wenig festgefügten Parteien und einer entsprechend schwach ausgeprägten Fraktionsdisziplin. Für die israelische Politik ist demgegenüber gerade die Kombination von ideologischer Polarisierung und fragmentiertem Mehrparteiensystem kennzeichnend. Die häufigen Partei- und Fraktionswechsel, die sich in permanenten Abspaltungen und Neugründungen niederschlagen und die israelische Parteienlandschaft zu einer der unübersichtlichsten unter allen westlichen Demokratien gemacht haben, stehen dem nicht entgegen. Sie ermöglichen zwar eine gewisse Flexibilität des parlamentarischen Abstimmungsverhaltens. Die Rückwirkungen dieses Verhaltens auf den Regierungsprozess gestalten sich aber eher destruktiv und sind mit dem parlamentarischen Prinzip überhaupt nur deshalb verträglich, weil die israelische Verfassungspraxis den Bestand einer Regierung nicht zwingend an deren Erfolgsbilanz im Parlament koppelt. Israel erinnert in dieser Beziehung eher an die lateinamerikanischen Staaten, wo die Verbin-

69 Vgl. z.B. Emanuele Ottolenghi, »Explaining Systemic Failure. The Direct Elections System and Israel's Special Elections of February 2001 « in: Israel Affairs 8 (2002) H.3, S. 144. 
dung von präsidentieller Regierungsform und Mehrparteiensystem ebenfalls große Probleme aufwirft und den entscheidenden Unterschied zum US-amerikanischen Prototyp des Präsidentialismus markiert. Die israelischen Verfassungsreformer sind insofern zu kurz gesprungen: Ein funktionierendes premier-parlamentarisches System hätte eine sehr viel konsequentere Umgestaltung der überkommenen Strukturen erfordert und über die Stärkung der präsidentiellen Komponente hinaus auch die Abkehr vom Verhältniswahlrecht mit einschließen müssen - also genau das, was man durch die Einführung der Direktwahl hatte umgehen wollen. Es scheint deshalb folgerichtig, wenn nach dem Scheitern des premier-parlamentarischen Experiments der alternative Pfad der Wahlrechtsreform (der in der Praxis auf eine Anhebung der Sperrklausel hinauslaufen dürfte) jetzt wieder verstärkt in den Blick gerät. Dies gilt zumal, als die Schaffung einer kodifizierten, die verstreuten »Grundgesetze« zusammenführenden Verfassung, die Gelegenheit zu einer solchen Reform böte, in Israel immer noch aussteht.

\section{Zusammenfassung}

Die 1992 beschlossene und 1996 erstmals durchgeführte Direktwahl des Premierministers in Israel stellt eines der interessantesten verfassungspolitischen Experimente der letzten Jahrzehnte dar. Mit der Direktwahl wurde der Versuch unternommen, eine richtiggehende Synthese zwischen der parlamentarischen und präsidentiellen Regierungsform, mithin ein »echtes « Mischsystem zu verwirklichen. Ziel der Reform war es, die Regierungsfähigkeit des Staates durch eine Aufwertung der institutionellen Position des Premierministers zu verbessern und diesen aus der notorischen Abhängigkeit von den kleineren Koalitionspartnern im zersplitterten israelischen Parteiensystem zu befreien. In der Praxis bewirkte die getrennte Abhaltung von Premier- und Parlamentswahlen freilich das Gegenteil. Indem die Wähler Gelegenheit erhielten, ihre Stimmen zu splitten, nahm die Fragmentierung des Parteiensystems weiter zu, sodass es für den Regierungschef noch schwieriger wurde, eine dauerhafte parlamentarische Unterstützung für seine Politik zu organisieren. Das Scheitern des präsidentiellen Parlamentarismus wäre auch bei einer Realisierung des ursprünglich geplanten radikaleren Entwurfs vorprogrammiert gewesen, der im parlamentarischen Beratungsprozess durch mehrere Korrekturen verwässert wurde. Die unintendierten Konsequenzen der Verfassungsreform führten dazu, dass der präsidentielle Parlamentarismus in Israel nur sieben Jahren Bestand hatte und das Land ab 2003 wieder zur rein parlamentarischen Regierungsweise zurückkehrte.

\section{Summary}

The direct election of the Prime Minister in Israel, which was introduced in the governmental system in 1992 and used for the first time in 1996, represents one of the most remarkable examples of constitutional engineering in Western democracies 
of the last decades. With direct election the reformers tried to put into practice a real synthesis between the parliamentary and presidential form of government, thus a »true" mixed system. The aim of the reform was to strengthen the governability of the state by revalorising the institutional position of the Prime Minister and making him less dependent from his coalition partners in the notoriously fragmented Israeli party system. However, in fact, the separation of the parliamentary and Prime Minister elections led to the opposite. Giving the voters the opportunity to split their votes, the system produced further fragmentation, thus making it even more difficult for the chief executive to organize a stable parliamentary majority for his policies. Presidential parliamentarism was condemned to failure, even if it had been introduced in the more radical way intended by the reformers in the first place and not been modified by the parties in the later political process. The unintended consequences of the reform were so striking, that the new system was abolished in 2003 after only seven years. By then Israel returned to the pure form of parliamentarism, which had been in operation until 1996.

\section{Aktuelle Neuerscheinung}

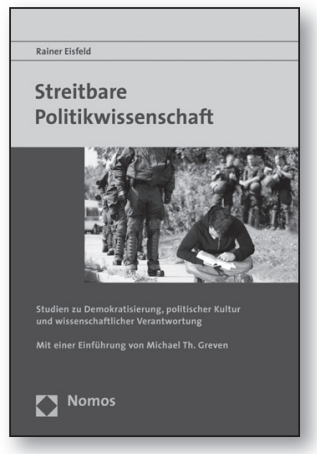

Streitbare

Politikwissenschaft

Studien zu Demokratisierung, politischer Kultur und wissenschaftlicher Verantwortung

Von Rainer Eisfeld

Mit einer Einführung von Michael Th. Greven

2006, 348 S., brosch., 49,- $€$, ISBN 3-8329-2050-1
Kritische - deshalb streitbare - Politikwissenschaft zeichnet sich durch ein historisches Verständnis ihres Gegenstandes aus. Sie betrachtet Politik nicht isoliert von gesellschaftlichen Prozessen. Sie untersucht gesellschaftliche Wirklichkeit mit der Zielsetzung erweiterter Beteiligungsrechte auf allen Ebenen und widmet Mechanismen der Herrschaftsausübung besondere Aufmerksamkeit.

Das Buch folgt diesen Grundregeln und beschäftigt sich mit den Themenbereichen des Pluralismus und der partizipatorischen Demokratie, Aspekten der politischen Kulturen Deutschlands, Englands und der USA im Vergleich, Portugals Transition von der Diktatur zur Demokratie, Zeitdiagnosen der Bundesrepublik Deutschland und schließlich mit zentralen Entwicklungen der deutschen Politikwissenschaft.

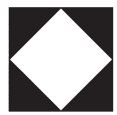

Nomos 